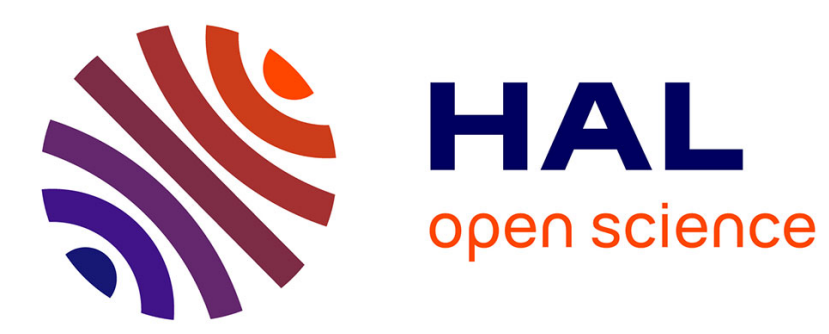

\title{
On higher-order boundary conditions at elastic-plastic boundaries in strain-gradient plasticity
}

\author{
Christian F. Niordson
}

\section{To cite this version:}

Christian F. Niordson. On higher-order boundary conditions at elastic-plastic boundaries in strain-gradient plasticity. Philosophical Magazine, 2009, 88 (30-32), pp.3731-3745. 10.1080/14786430802154823 . hal-00513902

\section{HAL Id: hal-00513902 \\ https://hal.science/hal-00513902}

Submitted on 1 Sep 2010

HAL is a multi-disciplinary open access archive for the deposit and dissemination of scientific research documents, whether they are published or not. The documents may come from teaching and research institutions in France or abroad, or from public or private research centers.
L'archive ouverte pluridisciplinaire HAL, est destinée au dépôt et à la diffusion de documents scientifiques de niveau recherche, publiés ou non, émanant des établissements d'enseignement et de recherche français ou étrangers, des laboratoires publics ou privés. 


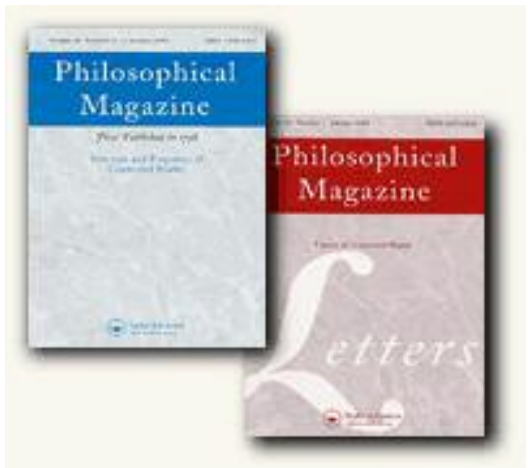

\section{On higher-order boundary conditions at elastic-plastic boundaries in strain-gradient plasticity}

\begin{tabular}{|c|c|}
\hline Journal: & Philosophical Magazine \& Philosophical Magazine Letters \\
\hline Manuscript ID: & TPHM-07-Dec-0377.R1 \\
\hline Journal Selection: & Philosophical Magazine \\
\hline $\begin{array}{l}\text { Date Submitted by the } \\
\text { Author: }\end{array}$ & 14-Apr-2008 \\
\hline Complete List of Authors: & $\begin{array}{l}\text { Niordson, Christian; Technical University of Denmark, Department } \\
\text { of Mechanical Engineering, Solid Mechanics }\end{array}$ \\
\hline Keywords: & mechanics, numerical modelling, plasticity \\
\hline Keywords (user supplied): & Size-effects, Strain gradient plasticity, Boundary conditions \\
\hline \multicolumn{2}{|c|}{$\begin{array}{l}\text { Note: The following files were submitted by the author for peer review, but cannot be converted } \\
\text { to PDF. You must view these files (e.g. movies) online. }\end{array}$} \\
\hline $\begin{array}{l}\text { bc.pstex } \\
\text { bc.pstex_t } \\
\text { bending.tex } \\
\text { bending.pstex } \\
\text { bending.pstex_t } \\
\text { bibitem.tex } \\
\text { conclusion.tex } \\
\text { deps_001.tex } \\
\text { deps_010.tex } \\
\text { deps_050.tex } \\
\text { eps_010.tex } \\
\text { eps_050.tex } \\
\text { external.tex } \\
\text { internal.tex } \\
\text { introduction.tex } \\
\text { main.tex }\end{array}$ & \\
\hline
\end{tabular}




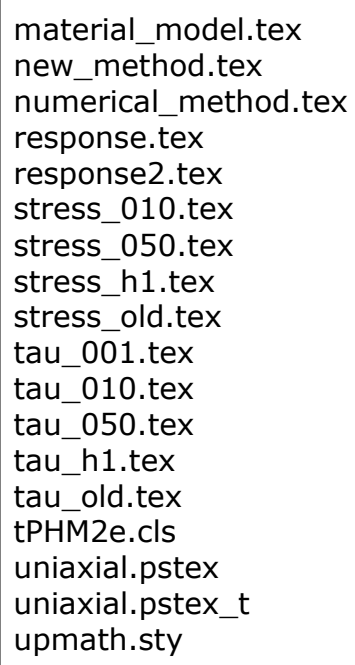

\section{ScholaroNE}




\title{
On higher-order boundary conditions at elastic-plastic boundaries in strain-gradient plasticity
}

\author{
Niordson, C. F* \\ Department of Mechanical Engineering, Solid Mechanics, Technical University of Denmark, \\ DK-2800 Kgs. Lyngby, Denmark
}

(April, 2008)

\begin{abstract}
A computational method for dealing with higher order boundary conditions on moving elastic-plastic boundaries in strain gradient plasticity is proposed. The basic idea is to skip the notion of a purely elastic regime, and instead introduce a pseudo-elastic regime, where plastic deformations are suppressed by using a very high artificial hardening modulus. Through numerical studies of pure bending under plane strain conditions, it is shown that this method predicts the build-up of higher order stresses in the pseudo-elastic regime. This has the effect of delaying the onset of incipient yield, as well as extending the plastic zone further toward the neutral axis of the beam, when compared to conventional models. Arguments supporting the present method are presented that rest on both mathematical and physical grounds. The results obtained are compared with other methods for dealing with higher order boundary conditions at internal elastic-plastic boundaries.
\end{abstract}

\section{Introduction}

Numerical analysis using the finite element method is now widely used for predicting size-dependent response as well as stress and strain fields in micron scale applications (see e.g. [1-5]). Several continuum models have been proposed to incorporate size-effects by using constitutive length-parameters. These models can be subdivided into two main groups: phenomenological models $[1,2,4,6,7]$ and crystal plasticity models [8-10]. The group of strain gradient plasticity models can also be subdivided into a group that is of lower order in nature, in that it retains the classical structure of a conventional boundary value problem [8] and a group that is of higher order nature and employs higher order stresses as work conjugates to strain gradients $[1,2,4,6,7,9,10]$. The latter group of models employs higher order boundary conditions in terms of strain (or slip) or work conjugate higher order tractions. The physical interpretation of these higher order boundary conditions has been the focus of much research $[2,9,11]$. In some visco-plastic formulations only higher order boundary conditions on external surfaces need to be specified. On the other hand, for higher order time-independent models, higher order boundary conditions must also be specified for internal elastic-plastic boundaries, and the appropriate choice here is far from obvious. In some numerical analyses simple assumptions are made for the conditions at moving internal boundaries [12], while others have employed advanced computational schemes in order to avoid specifying them [5].

In the present paper a simple computational method is proposed to overcome the problem of making specific choices for the boundary condition on internal elastic-plastic boundaries in time-independent strain gradient plasticity. Rather than having a distinct elastic regime, a pseudo-elastic regime is used below the yield limit, such that a negligible amount of plastic deformation takes place even here. Both mathematical and physical arguments supporting the assumption will be presented. The method is exemplified within the framework of the strain gradient plasticity theory of Fleck and Hutchinson [2], but the basic idea is applicable in other strain gradient models as well. The method is illustrated through numerical studies of pure bending under plane strain conditions.

\footnotetext{
${ }^{*}$ Corresponding author. Email: cn@mek.dtu.dk
} 


\section{Material Model}

An overview of the material model formulated by Fleck and Hutchinson [2] will be given in this section.

The total strain rate is taken as the sum of the elastic strain rate, $\dot{\epsilon}_{i j}^{E}$ and the plastic strain rate $\dot{\epsilon}^{P} m_{i j}$ :

$$
\dot{\epsilon}_{i j}=\dot{\epsilon}_{i j}^{E}+\dot{\epsilon}^{P} m_{i j}
$$

Here, $m_{i j}$ denotes the direction of the plastic strain rate, while $\dot{\epsilon}^{P}=\sqrt{\frac{2}{3} \dot{\epsilon}_{i j}^{P} \dot{\epsilon}_{i j}^{P}}$ is the rate of the conventional measure of effective plastic strain.

The strain gradient plasticity model can be based on the incremental work-functional

$$
\begin{array}{r}
I\left(\dot{u}_{i}, \dot{\epsilon}^{p}\right)=\frac{1}{2} \int_{V}\left(\left(\dot{\epsilon}_{i j}-\dot{\epsilon}^{p} m_{i j}\right) \mathcal{R}_{i j k l}\left(\dot{\epsilon}_{k l}-\dot{\epsilon}^{p} m_{k l}\right)+h\left[E^{p}\right] \dot{E}^{p^{2}}\right) d V \\
-\int_{S}\left(\dot{T}_{i} \dot{u}_{i}+\dot{t} \dot{\epsilon}^{p}\right) d S
\end{array}
$$

where the first term in the volume integral represents the elastic part of the work, and the second term represents the plastic expenditure of work, due to a gradient enhanced measure of effective plastic strain, $E^{p}$. Compared to the conventional effective plastic strain, this new measure of effective plastic strain is enriched with the three quadratic invariants of the gradient of the plastic strain rate, $\rho_{i j k}=\rho_{j i k}=\dot{\epsilon}_{i j, k}^{P}$, through the incremental relation

$$
\dot{E} P^{2}=\dot{\epsilon}^{2}+l_{1}^{2} I_{1}+4 l_{2}^{2} I_{2}+\frac{8}{3} l_{3}^{2} I_{3}
$$

Here, $I_{1}, I_{2}$ and $I_{3}$ are the three invariants of $\rho_{i j k}$, and $l_{1}, l_{2}$ and $l_{3}$ are three material length parameters, which are introduced for dimensional consistency. They define the length-scale on which size-effects become important.

A slight modification of the single parameter model by Aifantis [13], can be encompassed within this theoretical framework by using the following effective plastic strain definition

$$
\dot{E^{P}} \dot{P}^{2}=\dot{\epsilon}^{2}+l_{*}^{2} \dot{\epsilon}_{, i}^{P} \dot{\epsilon}_{, i}^{P}
$$

Here, $l_{*}$ is an alternative material length parameter.

The plastic strain increment is defined according to the usual relation for $J_{2}$ flow theory

$$
\dot{\epsilon}_{i j}^{P}=\frac{3}{2} \frac{s_{i j}}{\sigma_{(e)}} \dot{\epsilon}^{P}=m_{i j} \dot{\epsilon}^{P}
$$

where $s_{i j}$ is the deviator of the Cauchy stress $\sigma_{i j}$, the von Mises' effective stress is $\sigma_{(e)}=\sqrt{\frac{3}{2} s_{i j} s_{i j}}$, and $m_{i j}$ denotes the direction of the stress deviator.

Rewriting Equation (3) in terms of $m_{i j}$ and $\dot{\epsilon}^{P}$ results in

$$
\dot{E^{P^{2}}}=\dot{\epsilon}^{2}+A_{i j} \dot{\epsilon}_{, i}^{P} \dot{\epsilon}_{, j}^{P}+B_{i} \dot{\epsilon}_{, i}^{P} \dot{\epsilon}^{P}+C \dot{\epsilon}^{P^{2}}
$$

where the tensors $A_{i j}, B_{i}$ and $C$ depend on the three material length parameters $l_{1}, l_{2}$ and $l_{3}$ as well as on the spatial gradients of $m_{i j}$ (for details see [2]). For the single parameter model, $A_{i j}=l_{*}^{2} \delta_{i j}$, where $\delta_{i j}$ is Kronecker's delta, while $B_{i}=C=0$. 
Rendering the work functional stationary the principle of virtual work can be obtained as

$$
\int_{V}\left(\dot{\sigma}_{i j} \delta \dot{\epsilon}_{i j}+\left(\dot{q}-\dot{\sigma}_{(e)}\right) \delta \dot{\epsilon}^{P}+\dot{\tau}_{i} \delta \dot{\epsilon}_{, i}^{P}\right) d V=\int_{S}\left(\dot{T}_{i} \delta \dot{u}_{i}+\dot{t} \delta \dot{\epsilon}^{P}\right) d S
$$

where, $q$ is the work conjugate to the plastic strain, $\epsilon^{P}$, and $\tau_{i}$ are the higher order stresses, which are work conjugate to the effective plastic strain gradient. The right-hand side of the equation consists of the regular traction-displacement term in addition to a higher order traction term. Equation (7) shows how higher order boundary conditions need to be specified in addition to conventional traction displacement boundary conditions. The traction rate and higher order traction rate are given by the relations $\dot{T}_{i}=\dot{\sigma}_{i j} n_{j}$ and $\dot{t}=\dot{\tau}_{i} n_{i}$, respectively.

Since Equation (7) must hold for all sub-volumes, it shows that the effective plastic strain rate, $\dot{\epsilon}^{P}$, and the higher order traction rate $\dot{t}$ must be continuous over every boundary.

The constitutive equations are obtained as

$$
\begin{aligned}
\dot{\sigma}_{i j} & =\mathcal{R}_{i j k l}\left(\dot{\epsilon}_{k l}-\dot{\epsilon}^{P} m_{k l}\right) \\
\dot{q} & =h\left(\dot{\epsilon}^{P}+\frac{1}{2} B_{i} \dot{\epsilon}_{, i}^{P}+C \dot{\epsilon}^{P}\right) \\
\dot{\tau}_{i} & =h\left(A_{i j} \dot{\epsilon}_{, j}^{P}+\frac{1}{2} B_{i} \dot{\epsilon}^{P}\right)
\end{aligned}
$$

where $h=h\left[E^{p}\right]$ is the hardening modulus, and the isotropic elastic stiffness tensor is given by

$$
\mathcal{R}_{i j k l}=\frac{E}{1+\nu}\left(\frac{1}{2}\left(\delta_{i k} \delta_{j l}+\delta_{i l} \delta_{j k}\right)+\frac{\nu}{1-2 \nu} \delta_{i j} \delta_{k l}\right)
$$

where $E$ is Young's modulus and $\nu$ is Poisson's ratio.

The strong form of the equilibrium equations (consistent with the Principle of Virtual Work, Equation (7)) are

$$
\begin{aligned}
\dot{\sigma}_{i j, j} & =0 \\
\dot{q} & =\dot{\sigma}_{e}+\dot{\tau}_{i, i}
\end{aligned}
$$

The present strain gradient model is completed when the macroscopic uniaxial stress-strain curve is supplied together with the relevant length parameters for the specific framework used.

\section{Numerical Method}

Numerical solutions for pure plane strain bending will be presented using the finite element method described by Niordson and Hutchinson [12]. Special elements using 8 nodal degrees of freedom for each of the displacement components and 4 nodal degrees of freedom for the effective plastic strain proposed by Mikkelsen [14] are employed in a two-field finite element method similar to that used by de Borst and Mühlhaus [15], and de Borst and Pamin [16]. Further details on the numerical implementation can be found in $[12]$.

\section{External boundaries}

The principle of virtual work (Equation (7)) shows that higher order boundary conditions must be supplied in addition to the conventional boundary conditions. Referring to Figure 1, conventional tractiondisplacement boundary conditions must be imposed at the external surface, $S_{\text {ext }}$, of the body. The higher 


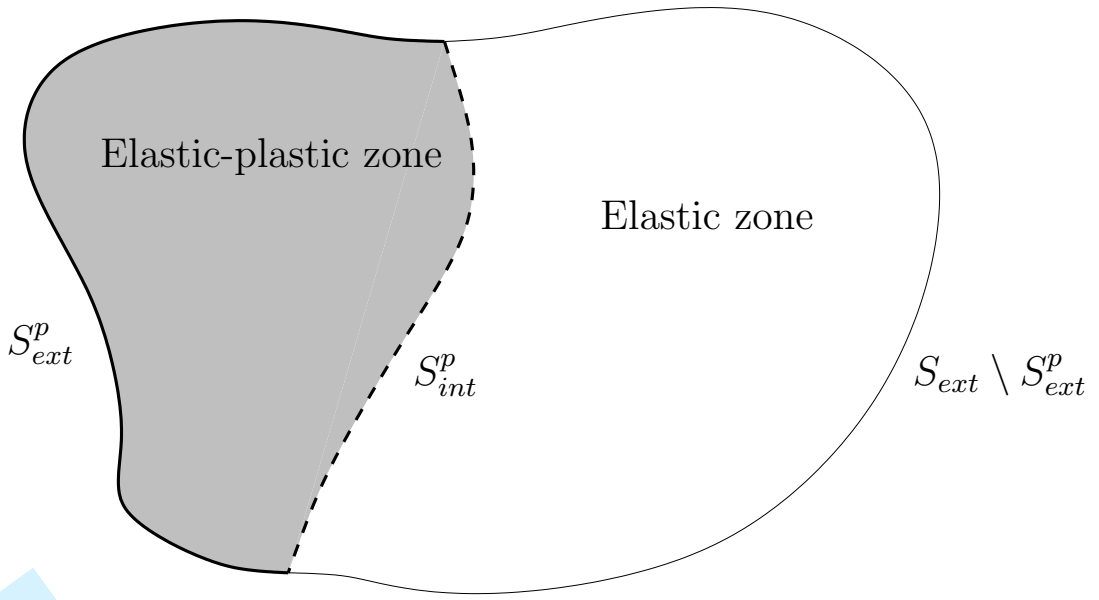

Figure 1. A schematic of a solid with relevant boundaries are shown. At the external surface, $S_{\text {ext }}$, conventional boundary conditions must be imposed. The boundary of the plastic zone, $S^{p}$ consists of an external part, $S_{\text {ext }}^{p}$ and an internal part $S_{\text {int }}^{p}$. Along $S^{p}$ higher order boundary conditions must be specified.

order boundary conditions must be specified only at the part of the boundary where the free variable, $\epsilon^{P}$, is active in the sense that we need to solve for it in order to solve the boundary value problem. We denote this boundary by $S^{p}$, and note that it consists of an external part, $S_{e x t}^{p}$ that is a part of $S_{\text {ext }}$, and an internal part $S_{i n t}^{p}$. We do not need to specify higher order boundary conditions at the boundaries of a purely elastic region.

One possibility for the higher order boundary condition at external boundaries is a full constraint on plastic flow, $\epsilon^{p}=0$, while another possibility is to impose the natural boundary condition, $t=0$. Intermediate boundary conditions can also be specified (see e.g. $[17,18]$ ).

\section{Internal boundaries}

In general internal boundaries exist between regions of the body with different microstructure (or orientation of the microstructure) or between elastic and elastic-plastic regions. A significant amount of work has been carried out in order to study the former type (see e.g. $[17,18]$ ). In the present work we do not consider this type of internal boundaries, which (mathematically) are merely a special case of external boundaries, even though their constitutive modeling is often more complex. Instead we focus at moving internal boundaries between elastic and elastic-plastic regions.

For internal elastic-plastic boundaries it is not obvious how to treat the higher order boundary conditions which must necessarily be specified between a purely elastic region and an elastic-plastic region. This problem has been discussed in the literature, and different suggestions have been put forward. A vanishing higher order traction rate $(\dot{t}=0)$ has been used in $[12,19,20]$. In [21] this boundary condition was compared to a full constraint on the plastic flow rate $\left(\dot{\epsilon}^{P}=0\right)$, and it was found that for the problem of plane strain sheet-necking, these two choices of higher order boundary conditions did not influence the results significantly. However, in general the specific choice of higher order boundary condition on internal elastic-plastic boundaries has an important effect on the predicted response of micron scale structures, as will be shown in Section 7.

The problematic nature of the internal higher order boundary conditions can be appreciated when studying Equation (13), which is the higher order equilibrium equation (or the consistency condition). For conventional plasticity the higher order traction vanishes and the equation reduces to $\dot{q}=\dot{\sigma}_{e}$. In this case no specific constitutive equation is needed for $q$. However, when we include strain gradient effects, the higher order equilibrium equation has the general form of Equation (13), and constitutive equations are needed for the higher order tractions $\tau_{i}$ as well as for $q$. The usual assumption is that $\tau_{i}$ vanishes in the elastic region. However, this is not obvious from the basic theoretical foundation of the Fleck-Hutchinson model, which is the incremental work functional (Eq. (2)). Furthermore, in most gradient dependent visco- 

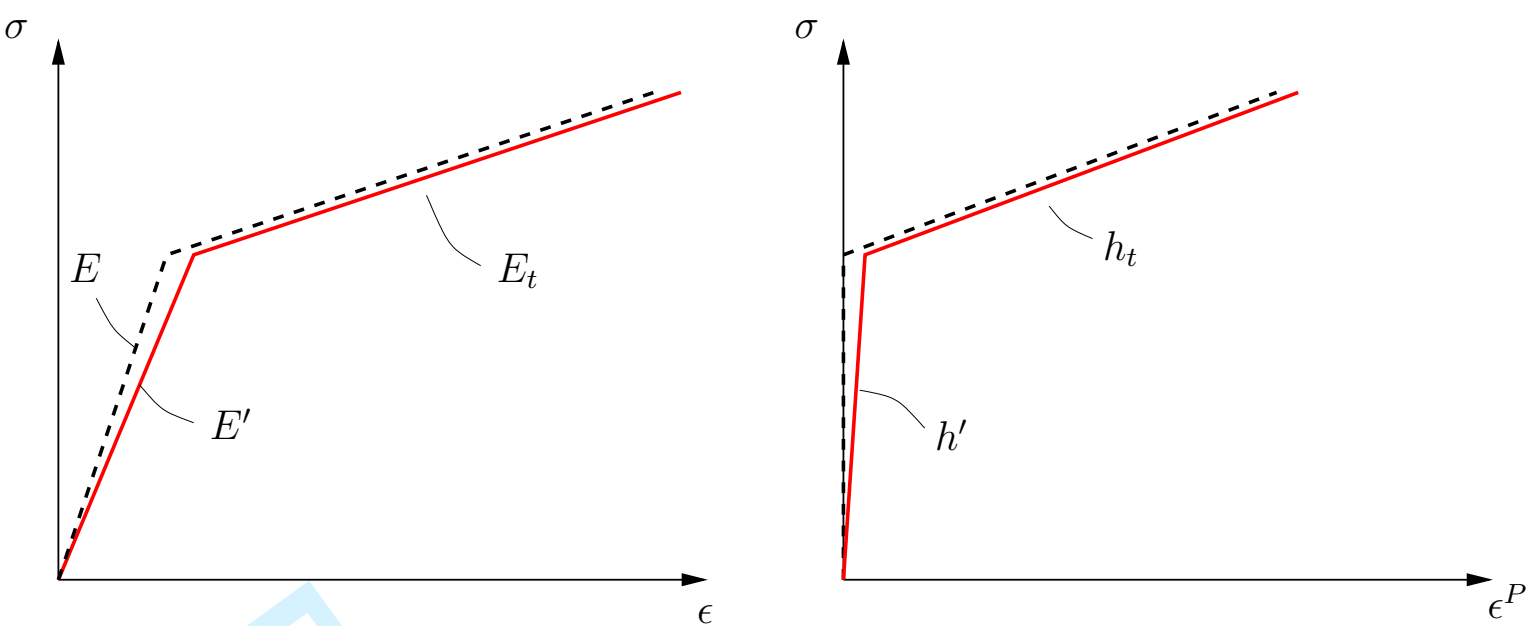

Figure 2. (a) Linearly hardening stress strain curve for a material with a purely elastic regime (dashed curve) and a pseudo-elastic regime (full curve). (b) Corresponding stress-plastic strain curves.

plastic models the higher order stresses do extend throughout the entire body regardless of the stress-level (see e.g. Borg [10]).

\section{Proposed method for dealing with internal boundary conditions}

In order to deal with the problem of internal boundary conditions, it is proposed to introduce a pseudoelastic regime in the uniaxial stress-strain curve, instead of a purely elastic regime. Within this regime a small but negligible amount of plastic dissipation takes place. The method is illustrated in Figure 2(a), where the dashed curve shows a uniaxial stress-strain curve with a purely elastic regime with a slope of Young's modulus $E$, and a linearly hardening elastic-plastic regime with the slope $E_{t}$. The full curve shows the modified uniaxial stress-strain curve where the purely elastic regime is replaced by a pseudo-elastic regime with the slope $E^{\prime}<E$. Figure 2(b) shows the corresponding uniaxial stress-plastic strain curve, where the hardening modulus is not defined for zero plastic strain, and the linear hardening plastic response gives rise to a constant value of the plastic hardening modulus $h_{t}$. The full curve shows the modification into a curve with a pseudo-elastic regime with a hardening modulus of $h^{\prime}$. By letting $h^{\prime}$ go to infinity plastic deformations in the pseudo-elasic regime are suppressed. In numerical calculations this is done by choosing $h^{\prime}$ significantly larger than Young's modulus $E$.

\section{$7 \quad$ Results for pure bending}

In order to illustrate the nature of the method proposed, pure bending under plane strain conditions is analyzed (see Figure 3). A beam of height $2 H$ is loaded with a pure bending moment per unit thickness $M$, which gives rise to the curvature of the beam, $\kappa$. A Cartesian coordinate system is embedded with the $x_{1}$-axis along the neutral axis and the $x_{2}$-axis perpendicular to the neutral axis in the plane of deformation.

Results will now be presented for beams with a ratio of the yield stress to Young's modulus of $\sigma_{y} / E=$ 0.004 , a Poisson's ratio of $\nu=0.3$ and a hardening modulus of $h_{t}=E / 10$. The beam is discretized using 500 elements through the half thickness of the beam, as symmetry around the neutral axis (due to the small strain assumption) is exploited. At the free surface a vanishing higher order traction is imposed for the higher order boundary condition. Following Peerlings [5] and Engelen et. al [22] a full constraint on the plastic strain is used at the neutral axis. In all the results presented, the single parameter model is used.

The first important question when evaluating the present method is the dependence of the predictions on the artificial hardening parameter $h^{\prime}$ in the pseudo-elastic regime. Figure 4(a) shows the stress distribution, $\sigma_{11}\left[x_{2}\right]$, through the upper half of the beam for increasing values of the surface strain $\kappa H$. For a conventional 


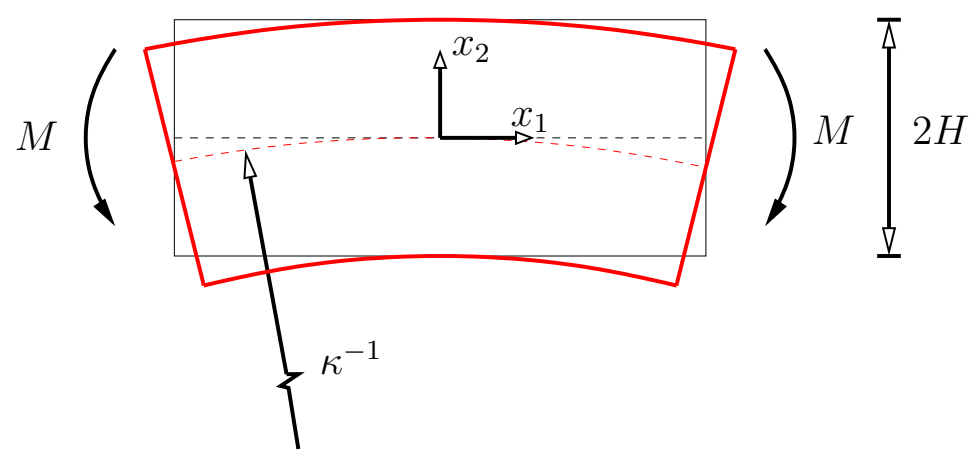

Figure 3. Beam under plane strain pure bending. The bending moment per unit thickness is $M$, and the curvature of the beam after deformation is $\kappa$. The beam height is $2 H$. A Cartesian coordinate system is embedded with the $x_{1}$-axis along the neutral axis and the $x_{2}$-axis perpendicular to the neutral axis in the plane of deformation.

material yielding initiates at the free surface for $\kappa H$ just above $0.4 \%$. The analyses are carried out for a moderate length parameter compared to the half beam thickness of $l_{*} / H=0.1$. For each value of the surface strain, results are shown for different values of $h^{\prime} / E ; 10,10^{2}, 10^{3}$ and $10^{6}$. The results suggest that the stress distribution converges to a limit value for $h^{\prime} / E \rightarrow \infty$, as the curves for $h^{\prime} / E \geq 10^{3}$ coincide. In general lower stresses are predicted for smaller values of $h^{\prime} / E$, as plasticity in the pseudo-elastic regime increases for decreasing $h^{\prime}$. For a conventional material the stress distribution in pure bending for a linearly hardening material is piecewise linear. On the other hand, for $l_{*} / H=0.1$, the Figure shows that the stress distribution is smooth, and has a positive curvature at the free surface $\left(x_{2} / H=1\right)$, so that the stress level here is increased over that of a conventional material. For large values of $h^{\prime} / E$ the plastic strain is inversely proportional to $h^{\prime}$. For $h^{\prime} / E=10^{6}$ the plastic strain level is on the order of $10^{-8}$, just before the onset of plasticity (defined as the point at which the beam surface enters the plastic regime) at the beam surface.

The higher order stress $\tau_{2}$ is shown in Figure 4(b) as a function of $x_{2}$ through the upper half of the beam, for different values of $h^{\prime} / E$, and at different surface strains, $\kappa H$. The Figure shows that the higher order stress increases with increasing deformation, and that it seems to converge for $h^{\prime} / E \rightarrow \infty$ as the curves coincide for $h^{\prime} / E \geq 10^{3}$. For the two lowest deformation levels $(\kappa H=0.2 \%$ and $0.4 \%)$, it is seen that there is a non-vanishing distribution of $\tau_{2}\left[x_{2}\right]$ even though the overall deformation is in the elastic range. This feature is different from other formulations of the Fleck and Hutchinson [2] strain gradient plasticity model as those suggested by [5,12,19-22], but it is consistent with the basic theoretical [5,12,19-22], but it is consistent with the basic theoretical foundation of the model as developed from the incremental work

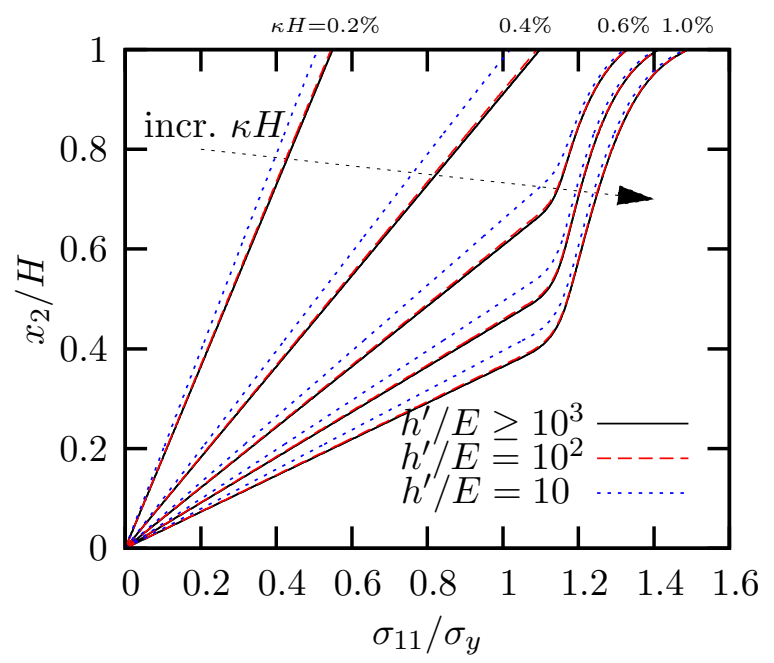

(a)

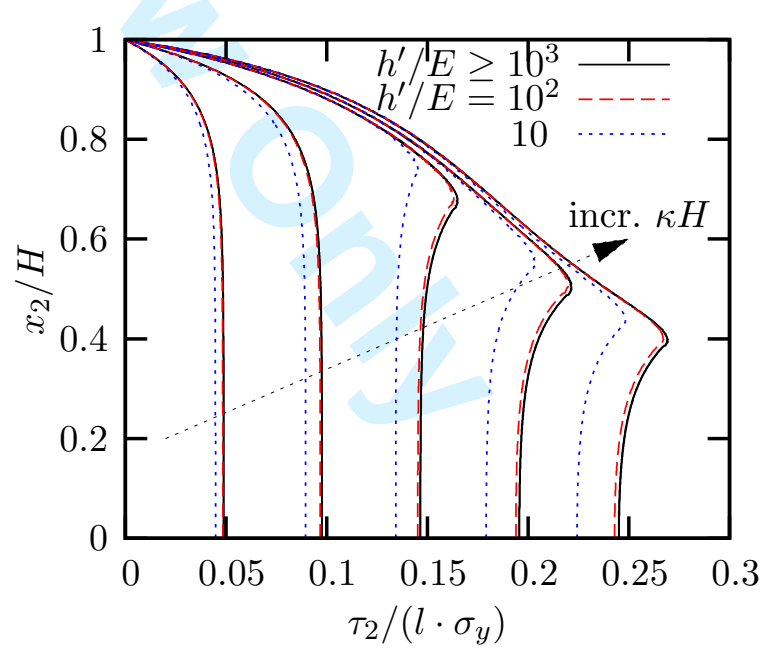

(b)

Figure 4. (a) Stress distribution and (b) higher order stress distribution through the upper half of the beam for different surface strains, $\kappa H$, and for different values of the artificial hardening parameter in the pseudo-elastic range. 


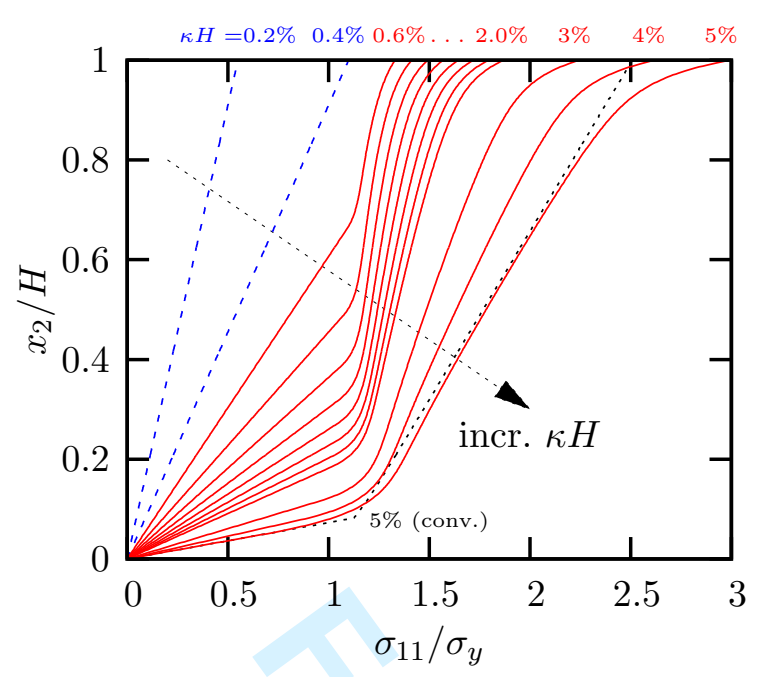

(a)

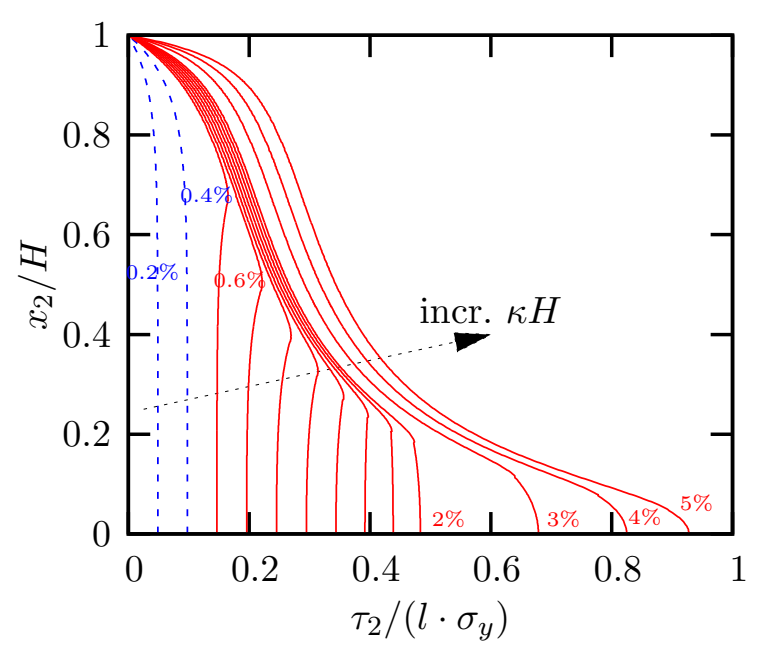

(b)

Figure 5. (a) Stress distribution and (b) higher order stress distribution for $l_{*} / H=0.1$ at different surface strains, $\kappa H$. The dashed curves show results for an overall loading before the onset of yield, while the full curves show results after the onset of yield.

functional (Equation (2)). In the results presented throughout the rest of this paper, a large value of the artificial hardening modulus, $h^{\prime} / E=10^{6}$, is used.

In Figures 5(a) and (b) results corresponding to those of Figures 4(a) and (b) are shown for $h^{\prime} / E=10^{6}$, and for surface strains $\kappa H$ up to $5 \%$. The dashed curves show results for overall loadings in the (pseudo-) elastic regime, and the full curves show results for overall deformations in the elastic-plastic regime. For comparison the stress distribution for a conventional material at an overall surface strain of $\kappa H=5 \%$ is shown in Figure 5(a) (dotted curve). It can be observed that for $l_{*} / H=0.1$ the stresses are enhanced over that of a conventional material near the surface. On the other hand, the stresses are smaller for $l_{*} / H=0.1$ when compared to conventional behaviour near the region where the elastic boundary is located for the conventional material. Figure 5(b) shows that for large deformation levels the higher order stress builds up at the neutral axis where a full constraint on plastic flow is imposed.

The increase in the stress near the surface for $l_{*} / H=0.1$ is a consequence of a suppression of plastic strain near the free surface due to the higher order boundary condition which demands zero higher order traction, and hence a vanishing gradient of effective plastic strain (according to Equation (10)) for the single parameter model. This is shown in Figure 6(a) where the plastic strain distributions are shown for different

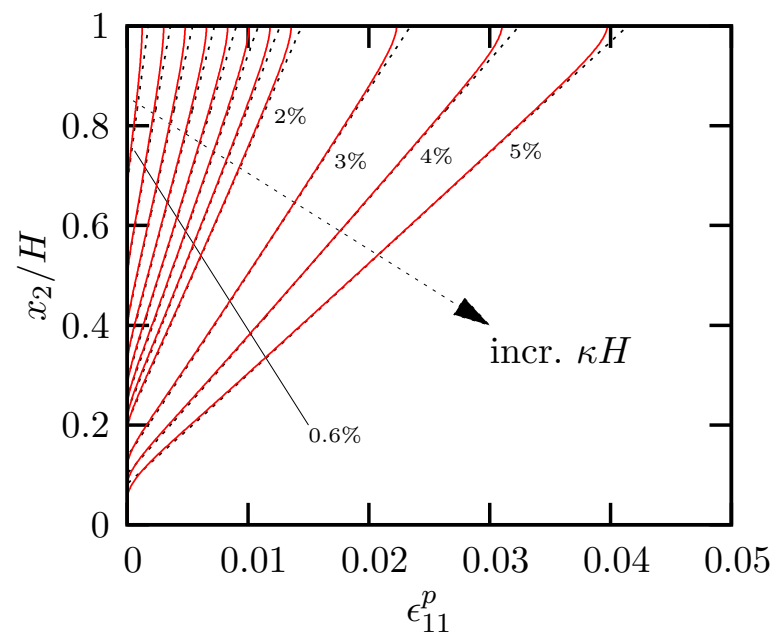

(a)

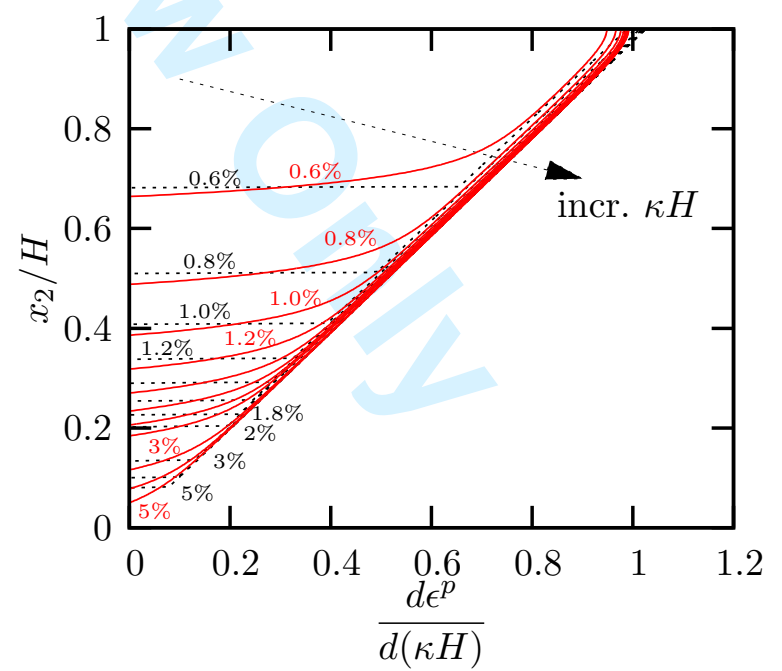

(b)

Figure 6. Profiles of (a) plastic strain and (b) effective plastic strain rate for $l_{*} / H=0.1$ at different surface strains, $\kappa H$. The full curves are the strain gradient results, while conventional results are shown for comparison (dotted curve). 


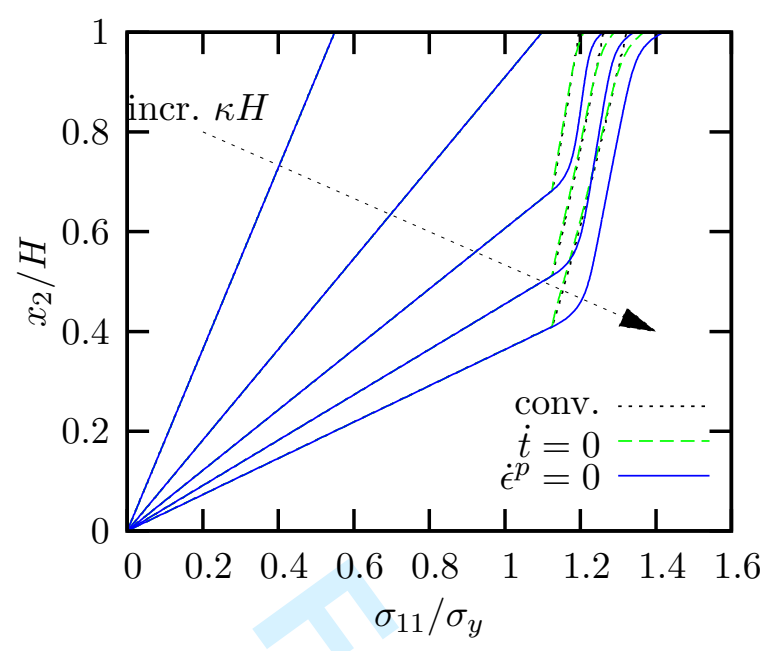

(a)

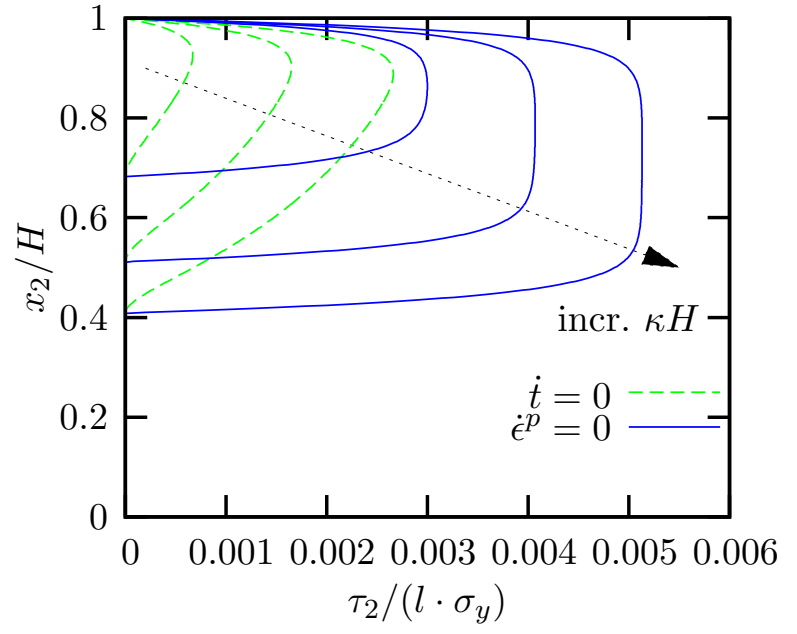

(b)

Figure 7. (a) Stress distribution and (b) higher order stress distribution through the upper half of the beam for the surface strains, $\kappa H=0.2 \%, 0.4 \%, 0.6 \%, 0.8 \%, 1.0 \%$. Results are shown for the incremental boundary condition $\dot{t}=0$ (dashed curves), the incremental boundary condition, $\dot{\epsilon}^{P}=0$ (solid curves). For comparison curves for a conventional material are also included (dotted curves).

values of the surface strain. The full curves show the results for $l_{*} / H=0.1$, while the dotted curves show corresponding results for a conventional material. Studying the curves for $\kappa H=5 \%$ a suppression of plastic strain near the surface and an enhancement of plastic strain near the elastic boundary for $l_{*} / H=0.1$ can be noticed. The distribution of the effective plastic strain rate is shown in Figure 6(b) for both $l_{*} / H=0.1$ and a conventional material. The jump in the plastic strain rate which is exhibited by the conventional material (due to the kink in the piecewise linear uniaxial response curve of Figure 2) is smoothed out for the gradient dependent material. The Figure also illustrates that the plastic region extends further toward the neutral axis for $l_{*} / H=0.1$ than for the conventional material, which gives rise to the stress decrease observed in Figure 5(a) near the elastic boundary for the conventional material.

In Figure 7 results are shown for the two different methods for treating higher order boundary conditions that are used in $[12,19-21]$. Here, a pure elastic regime is used, and the incremental boundary conditions, $\dot{t}=0$ or $\dot{\epsilon}^{P}=0$ are used on the internal moving elastic-plastic boundaries. The material length scale used is, for $l_{*} / H=0.1$. Results are shown for the overall deformation levels given by $\kappa H=0.2 \%, 0.4 \%, 0.6 \%, 0.8 \%, 1.0 \%$. The solid curves show results for the condition $\dot{\epsilon}^{P}=0$, the dashed curves show results for $\dot{t}=0$, while the dotted curves show conventional results included for comparison. The overall deformation levels correspond to those in Figures 4(a) and 4(b). It can be observed that a much smaller size effect is predicted for the boundary condition $\dot{t}=0$ than for $\dot{\epsilon}^{P}=0$. This is seen from both the stress distributions (Figure 7(a)), where the curves for $\dot{t}=0$ a quite close to those for the conventional material, and from Figure $7(\mathrm{~b})$, where is is seen that the higher order stress level is much smaller for $\dot{t}=0$ than for $\dot{\epsilon}^{P}=0$. The Figures also show that a much smaller size-effect is predicted using these two methods when compared to the method using the pseudo-elastic regime (see Figure 5). For the incremental boundary conditions the extent of the plastic zone is the same as for conventional plasticity, as the initial yield condition used is the conventional one.

Returning to the method proposed which employs the pseudo-elastic regime, results are shown for a much large value of the material length scale of $l_{*} / H=0.5$ in Figures $8(\mathrm{a})$ and (b), for different values of the surface strain. As in Figure 5, the dashed curves show results for overall loadings in the elastic range, while the full curves show results that are in the plastic range. For comparison a curve for a conventional material at a surface strain of $\kappa H=5 \%$ has been shown in Figure 8(a) (dotted curve). It is observed that for this larger value of the material length scale, the stress increase at the free surface over that of the conventional material is much larger than for $l_{*} / H=0.1$. Also, the decrease in stress near the neutral axis is much larger for this larger value of the material length scale. The distributions of higher order stress show that larger values of $\tau_{2}$ are obtained due to the larger value of $l_{*} / H$. An important difference between Figures $5(\mathrm{a})$ and $8(\mathrm{a})$ is that the curve for $\kappa H=0.6 \%$ is within the elastic range, with a linear stress 


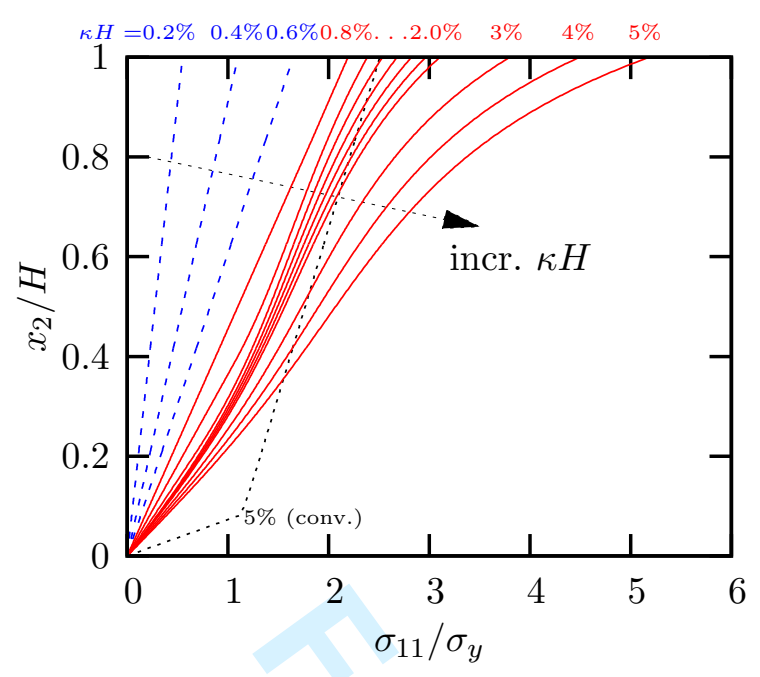

(a)

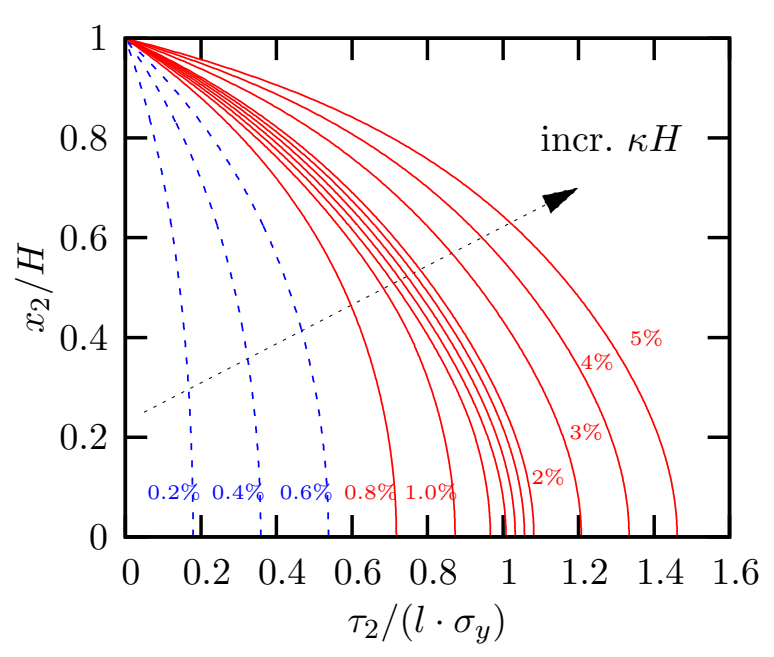

(b)

Figure 8. (a) Stress distribution and (b) higher order stress distribution for $l_{*} / H=0.5$ at different surface strains, $\kappa H$. The dashed curves show results for an overall loading before the onset of yield, while the full curves show results after the onset of yield.

distribution for $l_{*} / H=0.5$, while for $l_{*} / H=0.1$, plasticity has already set in. This can be explained by the negative value of $\tau_{i, i}=\tau_{2,2}$ at the free surface, which according to Equations (9) and (13) leads to a suppression of $\dot{\epsilon}^{P}$ in the purely pseudo-elastic regime. This suppression of $\dot{\epsilon}^{P}$ leads to a delay in the onset of plastic deformations beyond the pseudo-elastic regime.

For $l_{*} / H=0.5$ results corresponding to those in Figure 6 are shown in Figures 9(a) and (b). In Figure 9 (a) the difference in the plastic strain profiles is illustrated between the gradient dependent material (full curves) and the conventional material (dotted curves) for different overall strain values. Near the surface a significant suppression of plastic deformations is observed, while an enhancement of plastic deformation takes place near the neutral axis. The distribution of the plastic strain rate is shown in $9(\mathrm{~b})$, which illustrates how the jump in the plastic strain rate profile for a conventional material is smoothed out by strain gradient effects.

For a much smaller material length parameter relative to the half height of the beam, $l_{*} / H=0.01$, the distributions of the effective plastic strain rate and the higher order stress are shown in Figures 10(a) and (b), respectively. Even though there is a rather abrupt change in the plastic strain rate distribution, it is seen that it is still continuous. The effect of the vanishing higher order traction at the free surface still has

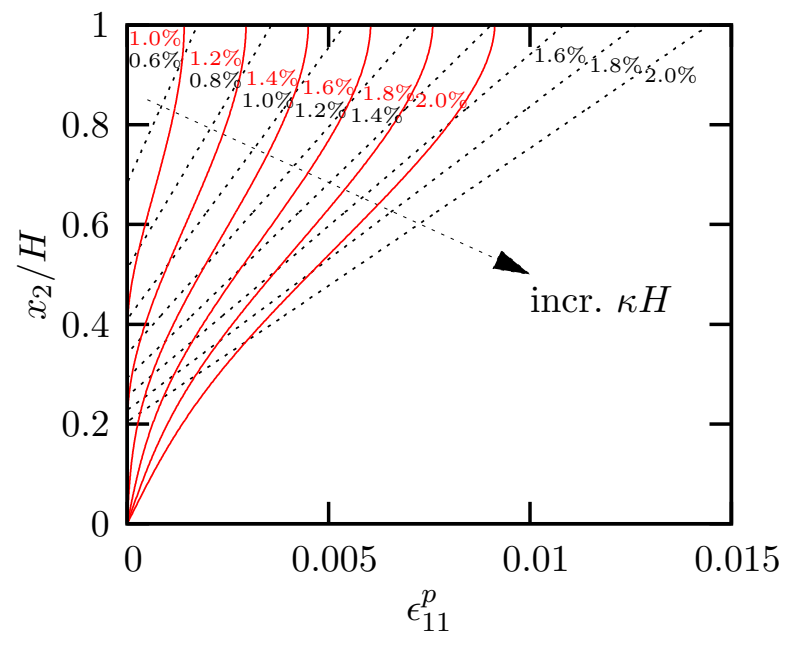

(a)

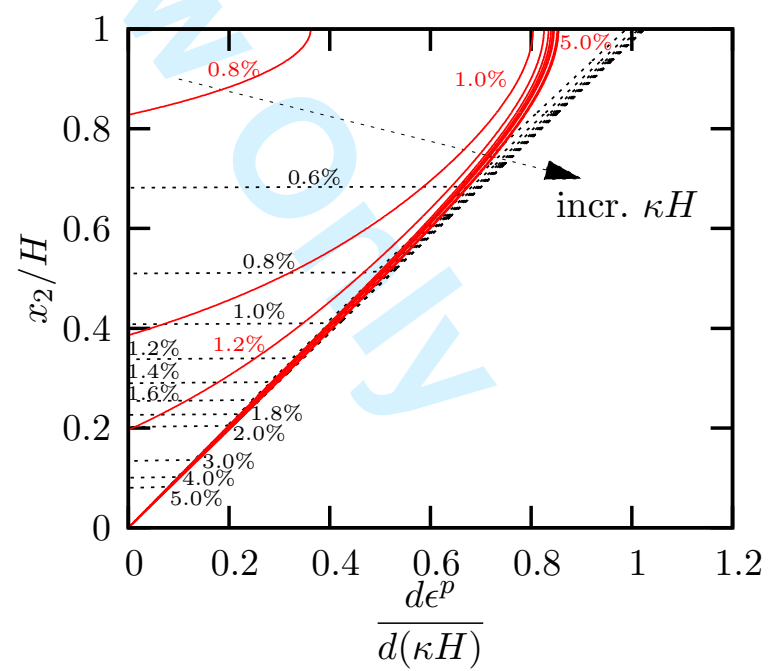

(b)

Figure 9. Profiles of (a) plastic strain and (b) effective plastic strain rate for $l_{*} / H=0.5$ at different surface strains, $\kappa H$. The full curves are the strain gradient results, while conventional results are shown for comparison (dotted curve). 


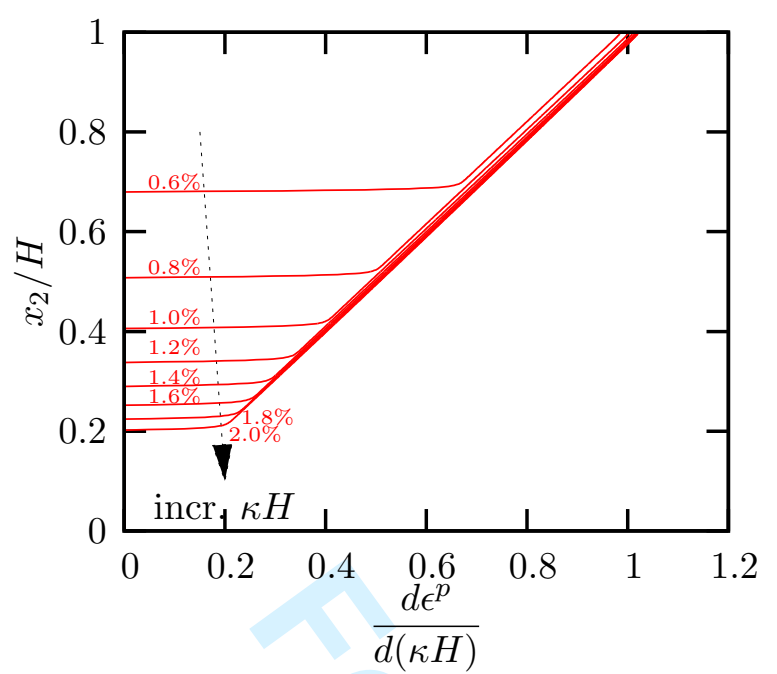

(a)

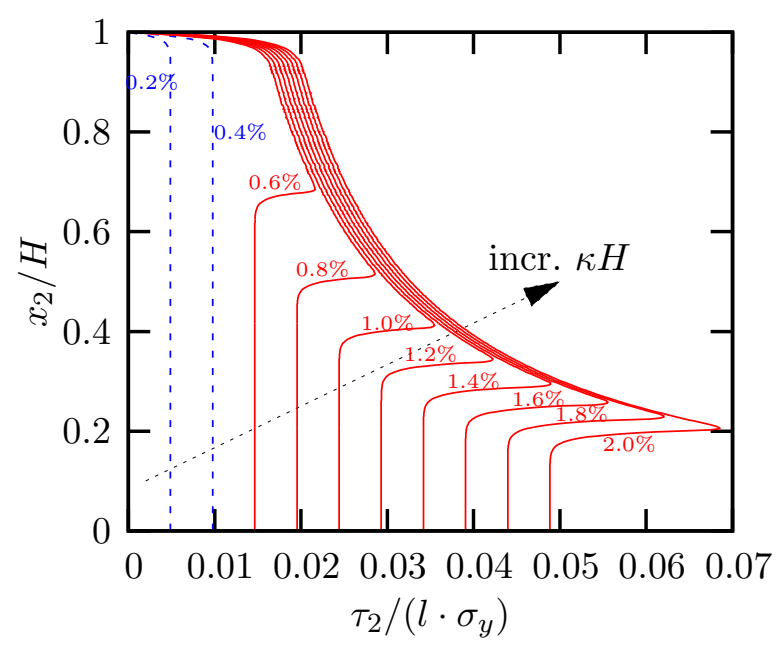

(b)

Figure 10. Profiles of (a) effective plastic strain rate and (b) higher order stress for $l_{*} / H=0.01$. The dashed curves show results for an overall loading before the onset of yield, while the full curves show results after the onset of yield.

the effect of producing zero slope of the plastic strain rate at this surface, even though it can not be seen from the Figure due to the very thin boundary layer. In Figure 10(b), the higher order traction is seen to build up to a constant level in the pseudo-elastic regime, well away from the free surface and the plastic boundary. This level can be shown analytically to be $l_{*}^{2} \sigma_{e, 2}$ for large values of $h^{\prime} / E$.

The overall response in terms of the normalized bending moment as a function of normalized curvature is shown in Figure 11(a), for the conventional material (dotted curve) and gradient dependent materials with different values of $l_{*} / H$. The bending moment (per unit thickness) is normalized by that at incipient yield for the conventional material, given by

$$
M_{y}=\frac{2 H^{2} \sigma_{y}}{3\left(1-\nu^{2}\right)}
$$

The Figure shows how the increase in the material length parameter leads to increased hardening, as well as a delay of incipient yield. The latter is different from many other formulations of the Fleck and Hutchinson model as those suggested by [5,12,19-22], where the higher order stresses do not build up in

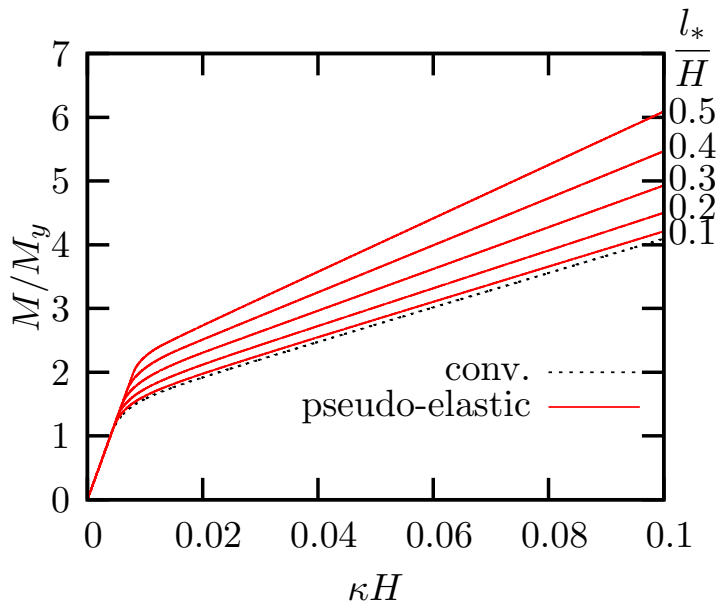

(a)

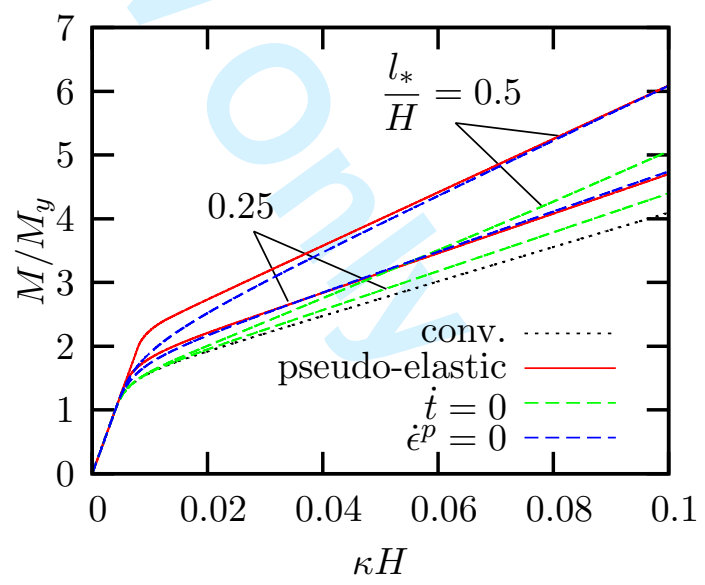

(b)

Figure 11. Overall response in terms of the normalized bending moment as a function of the normalized curvature, for a conventional material (dotted curve) and gradient dependent materials (full curves) with different values of $l_{*} / H$. 
a pseudo-elastic regime, and hence the onset of plastic deformation is independent of the material length scale. In Figure 11(b) response curves are compared for the different methods of dealing with higher order boundary conditions discussed in the present paper. Results are shown for the method using the pseudoelastic regime, as well as for the incremental boundary conditions $\dot{t}=0$ and $\dot{\epsilon}^{P}=0$. For comparison the conventional response curve is shown. The results illustrate that the two methods that employ incremental boundary conditions at the moving elastic-plastic boundary initially predict less hardening than the method using pseudo-elastic regime, as the latter predicts a delay of incipient yield. Furthermore, it is seen that the the boundary condition $\dot{t}=0$ gives significantly less hardening than the other two methods. For large deformation levels it is seen that the response curves are quite close for $\dot{\epsilon}^{P}=0$ and for the method employing the pseudo-elastic regime.

\section{Discussion}

A method for dealing with internal boundary conditions at elastic-plastic boundaries in strain gradient plasticity has been proposed. The method makes use of a pseudo-elastic regime in which a negligible amount of plastic dissipation takes place, rather than having a purely elastic regime. The pseudo-elastic regime is characterized by a very high hardening modulus, $h^{\prime}$, (compared to the Young's modulus of the material), which in the limit of going to infinity suppresses plastic deformation entirely in this regime. On the other hand, the higher order stresses approach a non-zero distribution, for $h^{\prime} \rightarrow \infty$, in the presence of a stress gradient. For pure bending under plane strain conditions this distribution can be expressed analytically by

$$
\tau_{2}\left[x_{2}, \kappa\right]=E \frac{\sqrt{1-\nu+\nu^{2}}}{1-\nu^{2}} l_{*}^{2}\left(1-\frac{\cosh \frac{x_{2}}{l}}{\cosh \frac{H}{l}}\right) \kappa
$$

before the initiation of plastic deformations. Under plane stress conditions the term involving Poisson's ratio, $\nu$, should be excluded. This build up of higher order stresses in the pseudo-elastic regime has three important implications due to the appearance of the higher order stress term in Equation (13)

- The onset of plastic deformations is delayed.

- At later stages of deformation the plastic zone extends further towards the neutral axis when compared to conventional material behavior.

- The plastic zone will reach the neutral axis for a finite curvature, $\kappa$.

It should be noted that the two latter points are also valid for the analyses of Peerlings [5] and Engelen et. al $[22]$.

The method presented here has one other important feature, namely that it insensitive to small variations in the macroscopic uniaxial stress-strain curve which is used as constitutive input to the Fleck and Hutchinson model. More specifically, to this author's knowledge, all other approaches used for dealing with higher order boundary conditions at internal boundaries (including $[5,12,19-22]$ ) are sensitive to the exact definition of the yield point, since this point defines the point at which higher order stresses start to build up. Since the specification of a yield point is a matter of definition in the present model, due to the introduction of the pseudo-elastic regime, as it also is for real materials, it has no effect on the predictions of the model.

It is interesting to note that the use of the Ramberg-Osgood curve for the uniaxial stress-strain curve (as used by Fleck and Hutchinson [2]) is consistent with the basic ideas brought forward in the present paper, as this curve does not have a distinct elastic regime, and hence higher order stresses will build up from the onset of deformation.

It is shown through comparison with methods that employ incremental boundary conditions at elasticplastic boundaries [12,19-21], that initially a much larger size-effect is predicted when using the pseudoelastic regime. This is due to the build up of higher order stresses in the pseudo-elastic regime, which results in an enhancement of size-effects when compared to methods where higher order stresses only 
builds up in the plastic regime. At larger deformation levels the response curves for the incremental boundary condition, $\dot{\epsilon}^{P}=0$, are close to those for the pseudo-elastic regime. This can be explained from the fact that the elastic-plastic boundary extends very slowly for the incremental boundary condition, as it approaches the neutral axis of the beam, and for the two different methods the incremental problem are almost the same, as one imposes $\dot{\epsilon}^{P}=0$ at the neutral axis, while the other imposes the same boundary condition at the elastic-plastic boundary which is almost at a stand-still near the neutral axis.

The development of higher order stresses in the pseudo-elastic regime might seem to be at odds with the strain gradient plasticity theory proposed by Fleck and Hutchinson [2]. However, the basic idea in this theory is that plastic work is expended due to the development of the gradient enhanced measure of plastic strain, $E^{p}$ (see Equations (3) and (4)). The requirement of stationarity of the second-order incremental work-functional defined in Equation (2), is the tool used for obtaining equilibrium conditions and constitutive equations, for a material where the second order plastic work density is given by

$$
h\left[E^{p}\right] \dot{E}^{p^{2}}
$$

Now consider an elastic-perfectly plastic material for which the hardening modulus $h$ must vanish in the plastic regime. For this material the only way of accounting for increased dissipation due to a plastic strain gradient, manifesting itself in the theory through the fact that $\dot{E}^{p} \neq \dot{\epsilon}^{p}$, is if higher order stresses can develop prior to entering the plastic regime. Hence, the development of higher order stresses prior to entering the plastic regime is not in conflict with the strain gradient plasticity theory by Fleck and Hutchinson [2], and even though the method proposed here does not follow the original development of the theory based on the second order work functional alone, it seems to be a natural extension supporting the basic principle underlying the theory.

In this paper pure bending under a monotonically increasing load is analyzed as a test example for the method proposed. The method, however, is general and can be employed for problems involving nonmonotonic loading as well. In this case the same pseudo-elastic regime can be used during elastic unloading. This will lead to higher order stresses that change during unloading, as they have been shown to do under the initial elastic deformation.

\section{References}

[1] Fleck, N. A. and Hutchinson, J. W., in Advances in Applied Mechanics 33, edited by J. W. Hutchinson and T. Y. Wu (Academic Press, 1997), pp. 295-361.

[2] Fleck, N. A. and Hutchinson, J. W., J. Mech. Phys. Solids 492245 (2001).

[3] Huang, Y. and Gao, H. and Nix, W.D. and Hutchinson, J. W., J. Mech. Phys. Solids 4899 (2000).

[4] Legarth, B.N., Modelling and Simulation in Materials Science and Engineering 15 S71 (2007).

[5] Peerlings, R. H. J., Modelling and Simulation in Materials Science and Engineering 15 S109 (2007).

[6] Gao, H. and Huang, Y. and Nix, W.D. and Hutchinson, J. W., J. Mech. Phys. Solids 471239 (1999).

[7] Gudmundson, P., J. Mech. Phys. Solids 521379 (2004).

[8] Acharya, A. and Bassani, J. L., in Plastic and Fracture Instabilities in Materials, edited by N. Ghoniem (ASME, New York), pp. 75-80, 1995.

[9] Gurtin, M. E., J. Mech. Phys. Solids 505 (2002).

[10] Borg, U., Int. J. Plasticity 231400 (2007).

[11] Gurtin, M.E. and Needleman, A., J. Mech. Phys. Solids 531 (2005).

[12] Niordson, C. F. and Hutchinson, J. W., International Journal for Numerical Methods in Engineering 56961 (2003).

[13] Aifantis, E. C., Transactions of the ASME. Journal of Engineering Materials and Technology 106326 (1984).

[14] Mikkelsen, L.P., Proc. from the 2007 ABAQUS Users' Conference, 482 (2007).

[15] de Borst, R. and Mühlhaus, H.-B., International Journal for Numerical Methods in Engineering 35521 (1992).

[16] de Borst, R. and Pamin, J., International Journal for Numerical Methods in Engineering 392477 (1996).

[17] Aifantis, K.E. and Willis, J.R., J. Mech. Phys. Solids 531047 (2005).

[18] Borg, U. and Fleck, N. A., Modelling and Simulation in Materials Science and Engineering 15 S1 (2007).

[19] Niordson, C. F., J. Mech. Phys. Solids 511863 (2003).

[20] Niordson, C. F. and Tvergaard V., J. Applied Mechanics 73246 (2006).

[21] Niordson, C. F. and Redanz, P., J. Mech. Phys. Solids 522431 (2004).

[22] Engelen, R.A.B. and Fleck, N.A. and Peerlings, R.H.J. and Geers, M.G.D., International Journal of Solids and Structures 431857 (2006). 


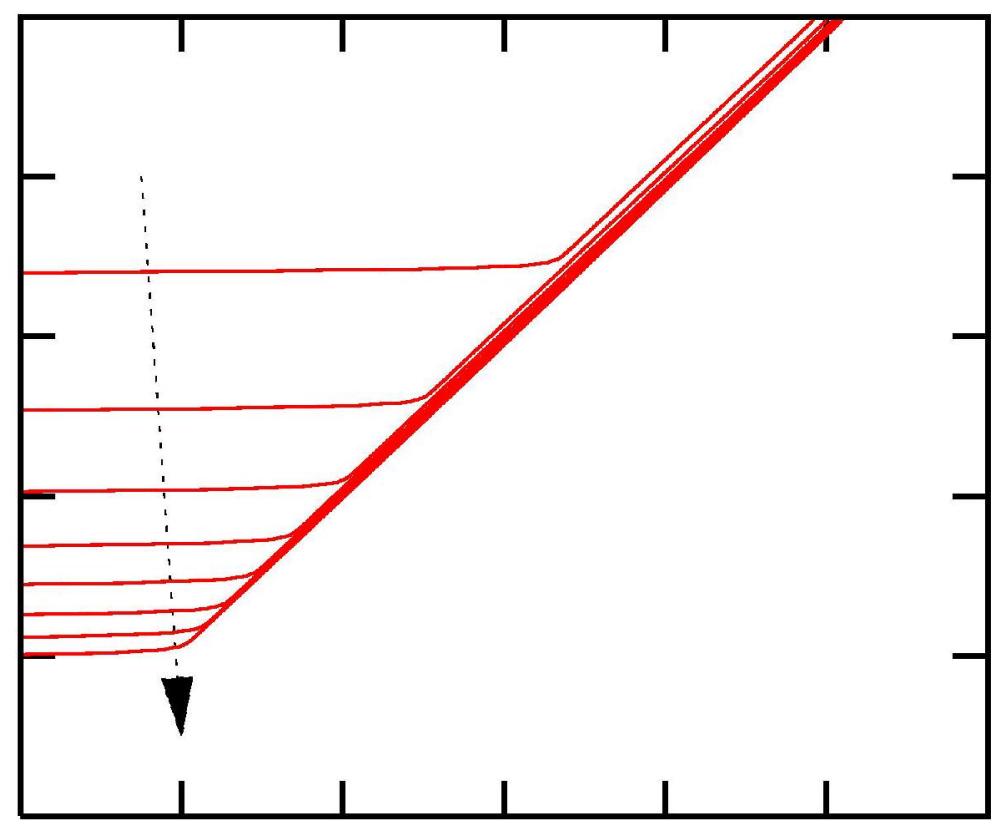

$82 \times 64 \mathrm{~mm}(600 \times 600 \mathrm{DPI})$ 


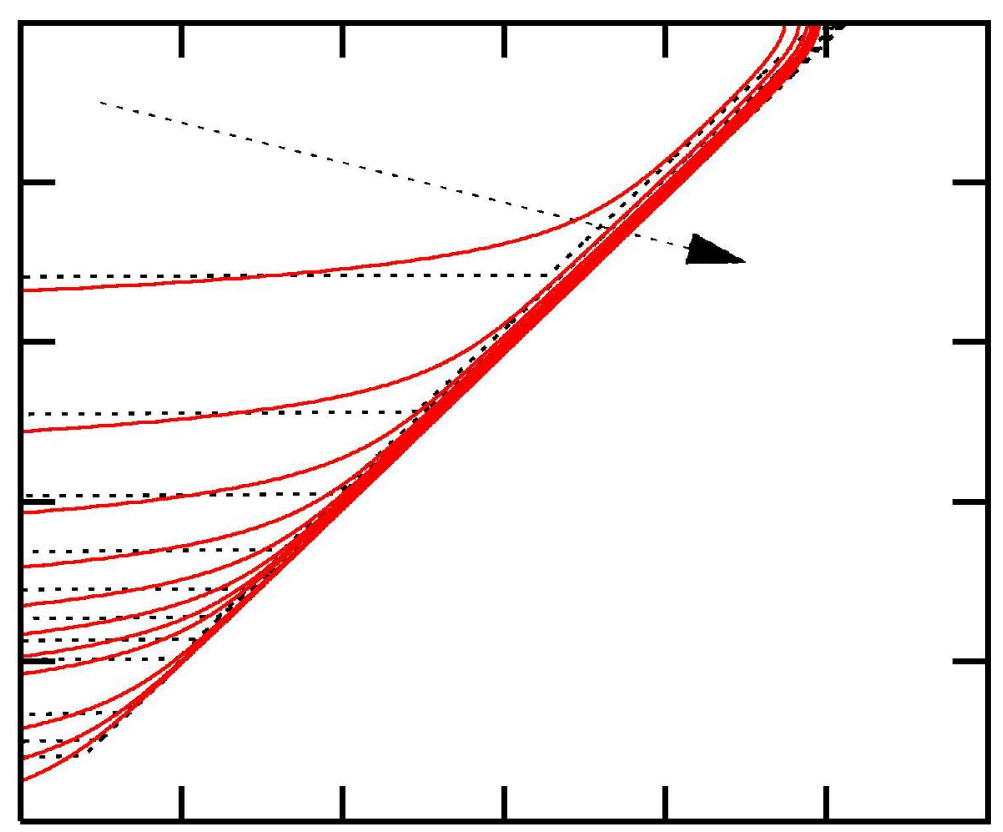

$82 \times 64 \mathrm{~mm}(600 \times 600 \mathrm{DPI})$ 


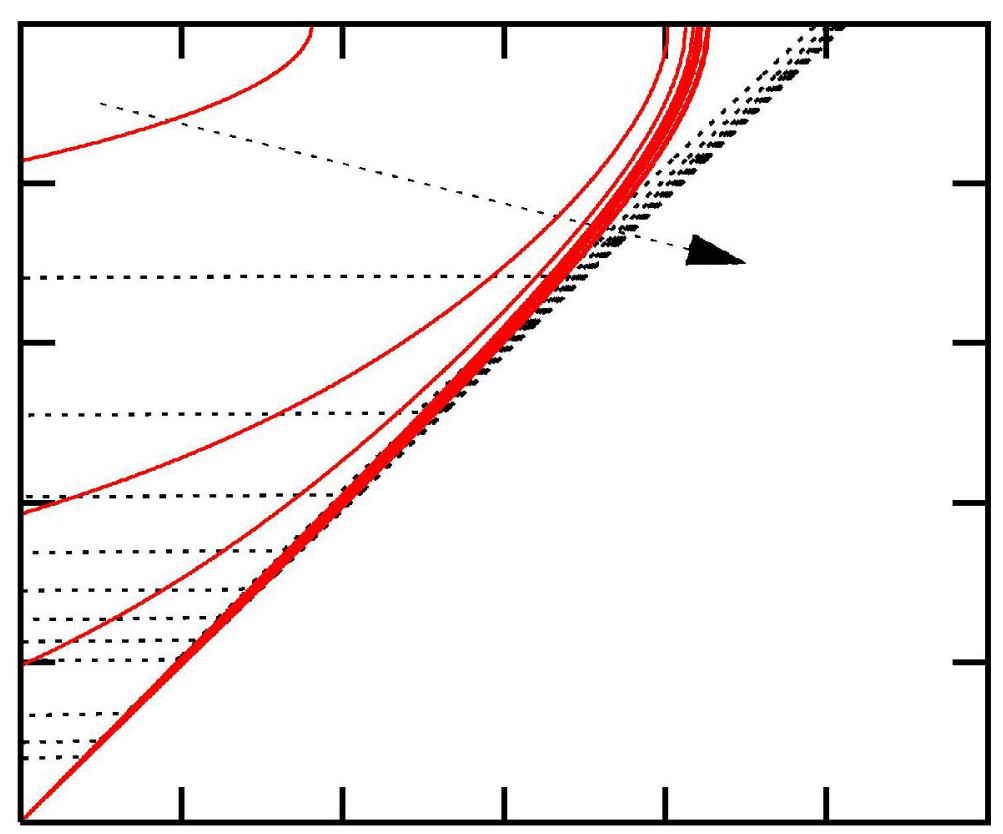

$82 \times 64 \mathrm{~mm}(600 \times 600 \mathrm{DPI})$ 


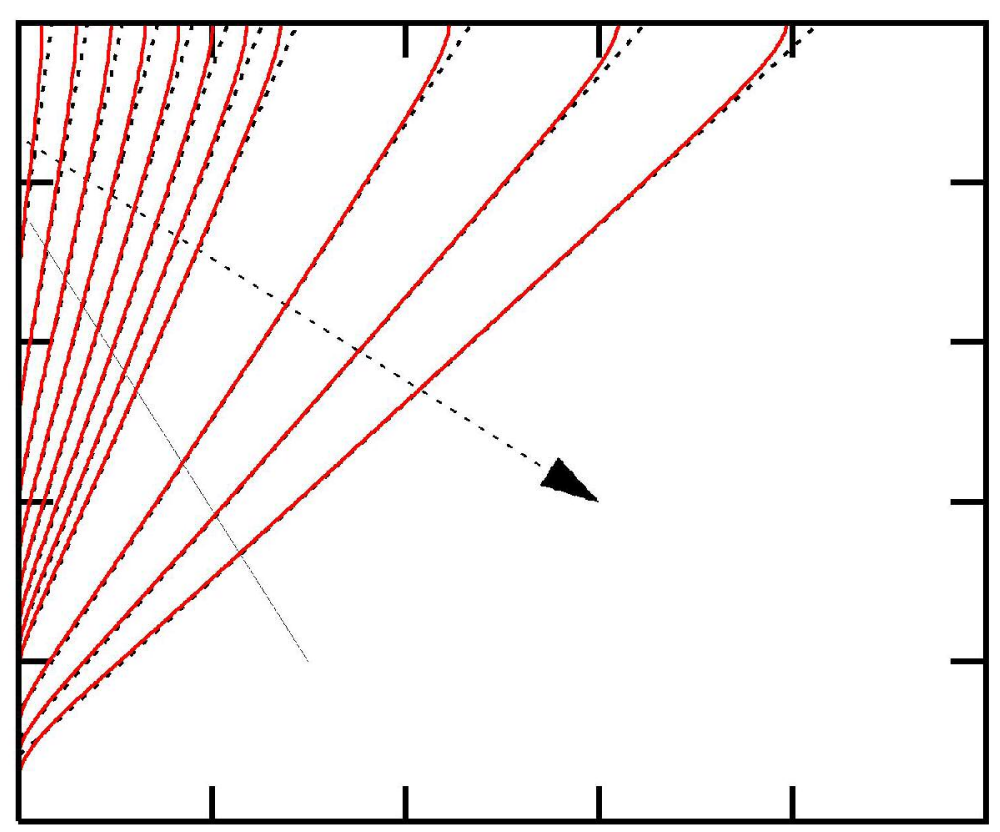

$82 \times 64 \mathrm{~mm}(600 \times 600 \mathrm{DPI})$ 


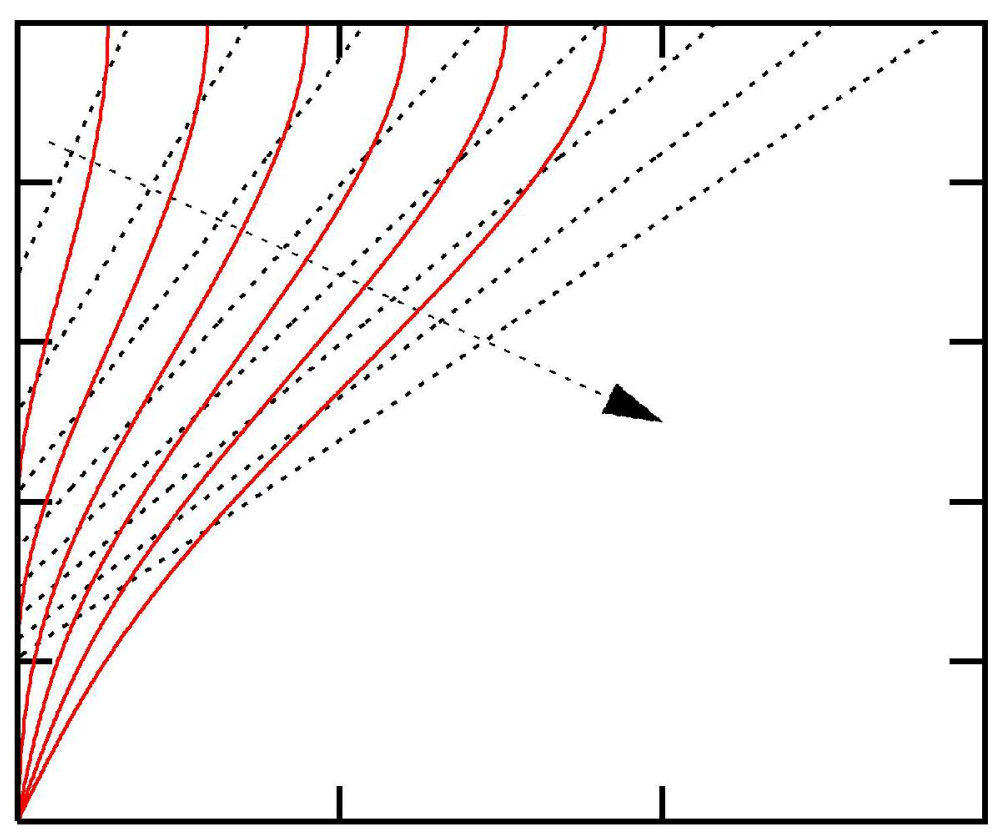

$82 \times 64 \mathrm{~mm}(600 \times 600 \mathrm{DPI})$ 


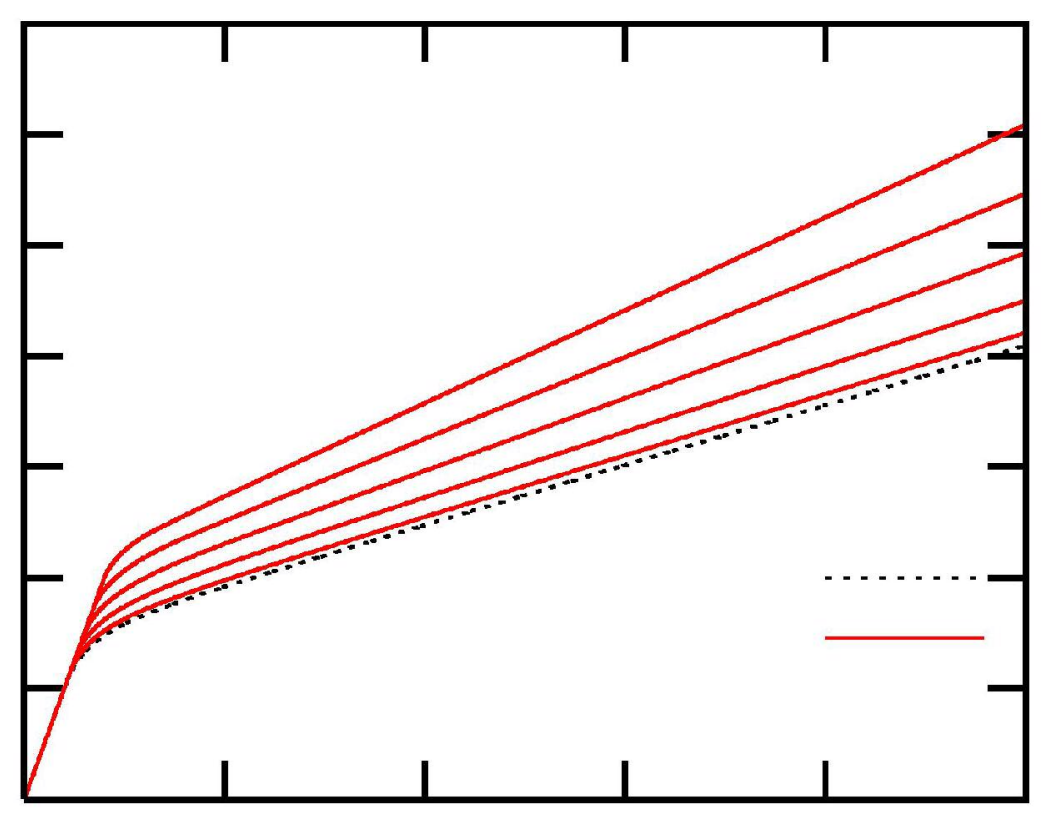

$76 \times 59 \mathrm{~mm}(600 \times 600 \mathrm{DPI})$ 


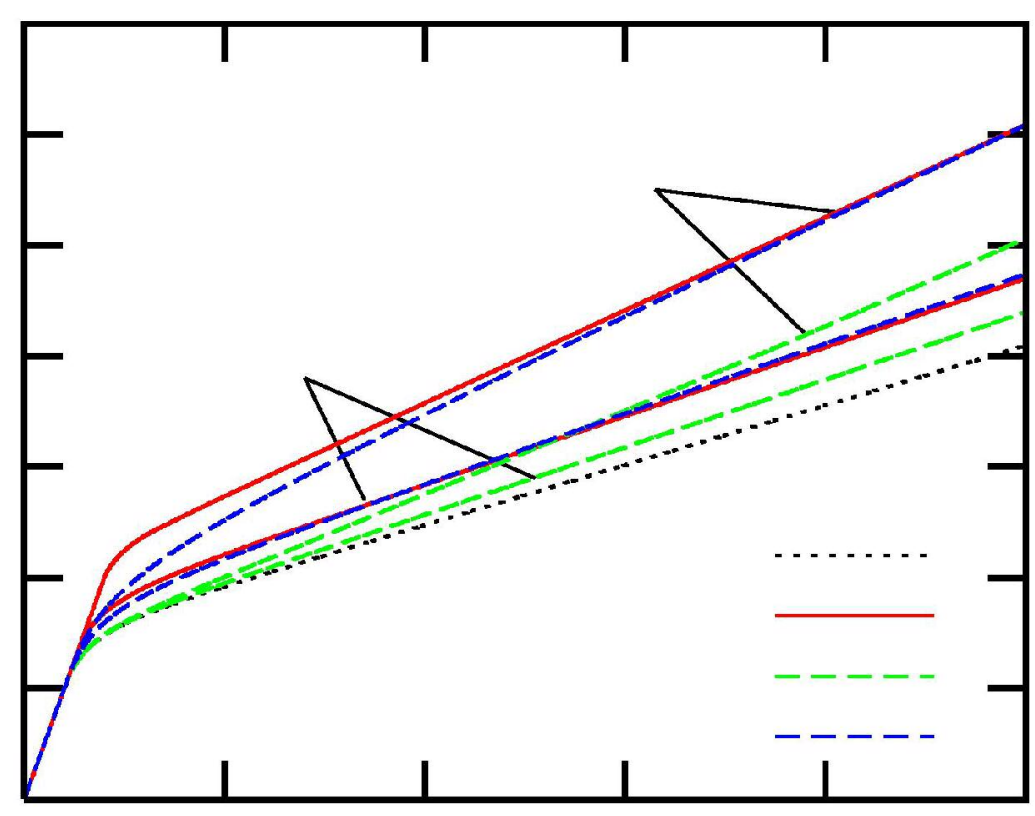

$76 \times 59 \mathrm{~mm}(600 \times 600 \mathrm{DPI})$ 


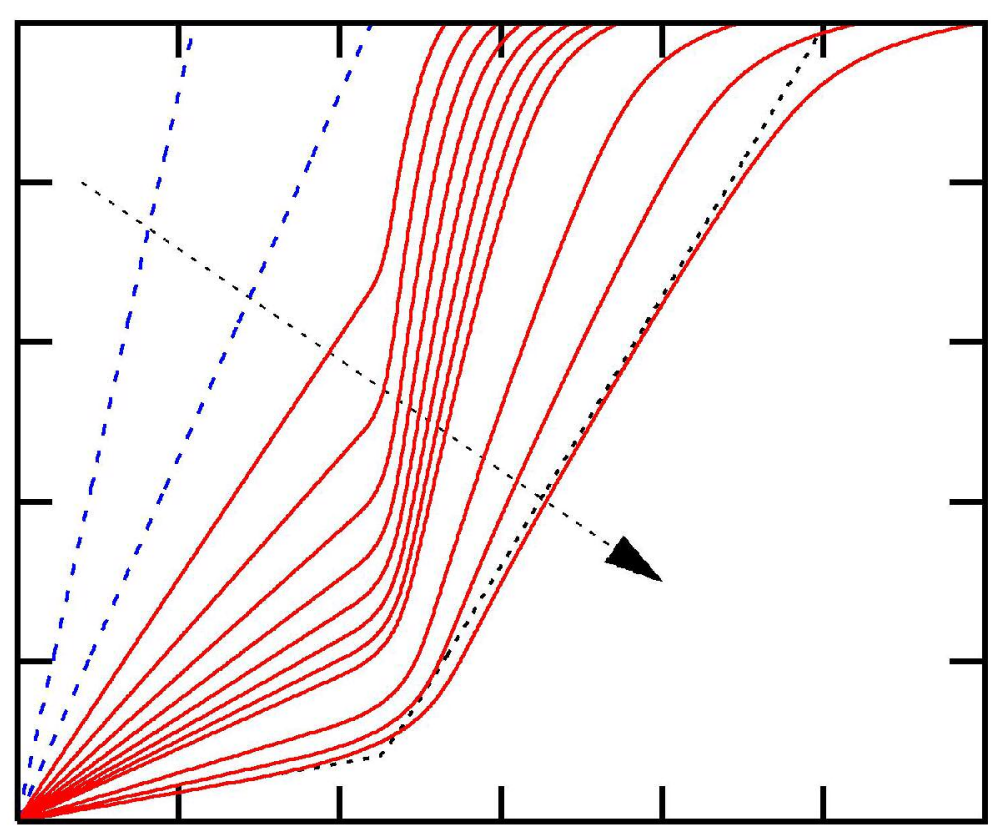

$82 \times 64 \mathrm{~mm}(600 \times 600 \mathrm{DPI})$ 


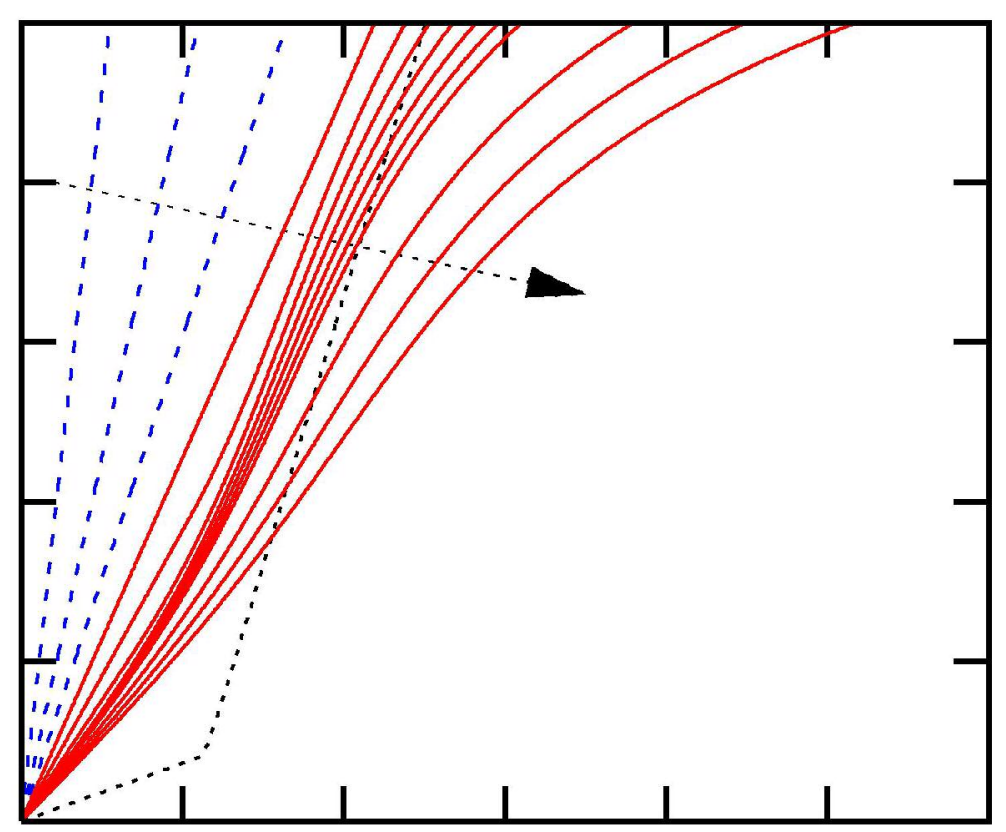

$82 \times 64 \mathrm{~mm}(600 \times 600 \mathrm{DPI})$ 


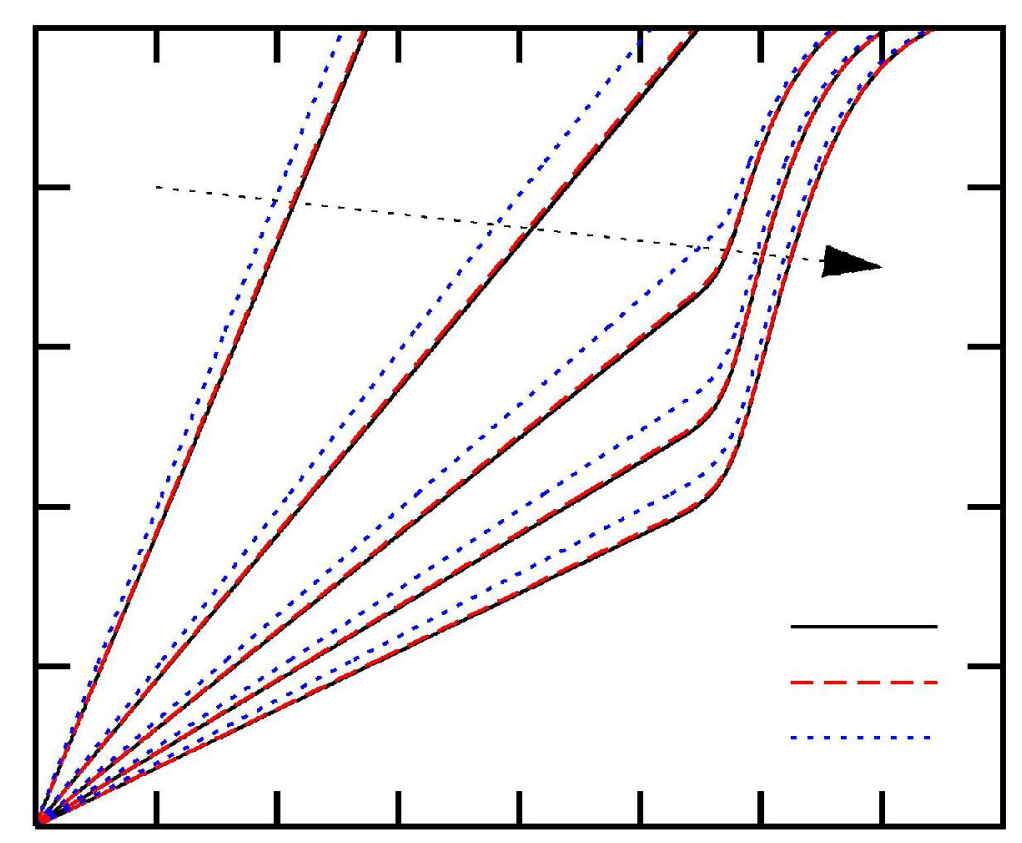

$82 \times 64 \mathrm{~mm}(600 \times 600 \mathrm{DPI})$ 


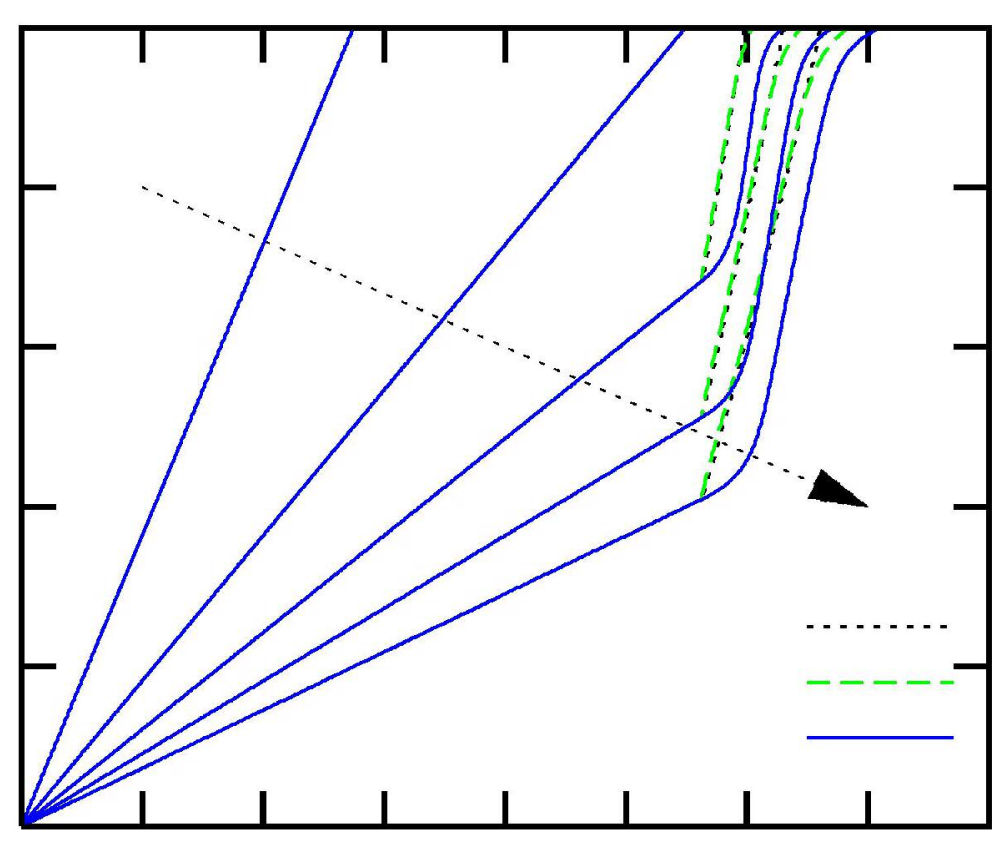

$82 \times 64 \mathrm{~mm}(600 \times 600 \mathrm{DPI})$ 


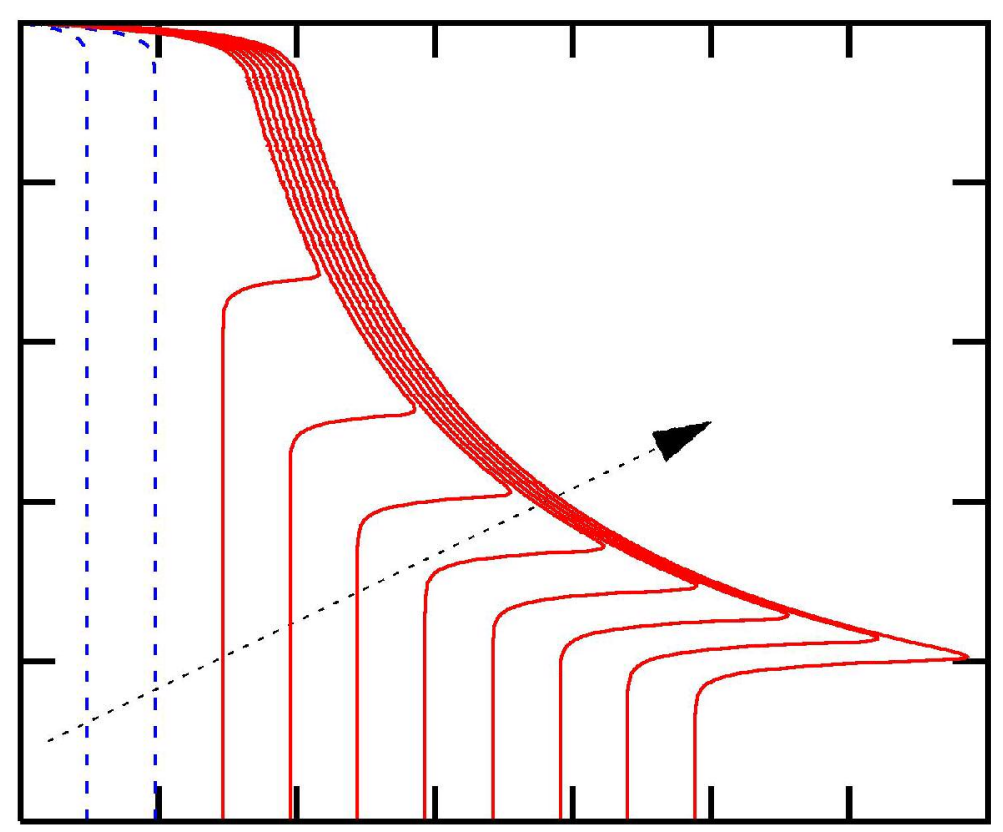

$82 \times 64 \mathrm{~mm}(600 \times 600 \mathrm{DPI})$ 


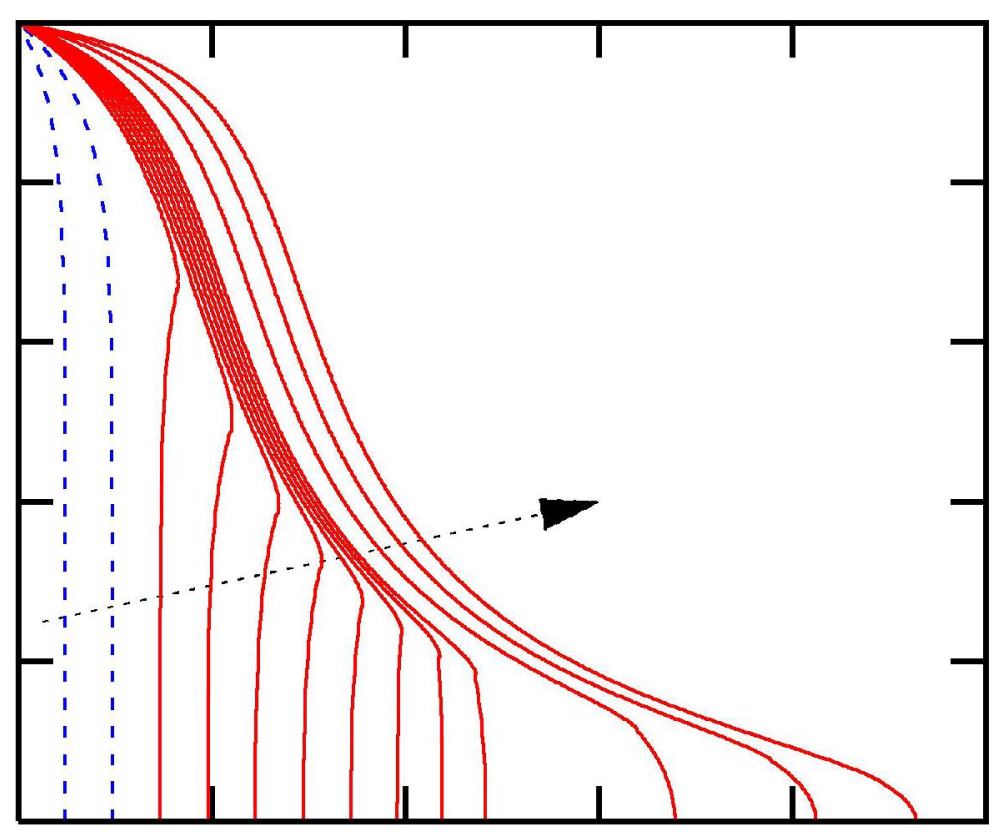

$82 \times 64 \mathrm{~mm}(600 \times 600 \mathrm{DPI})$ 


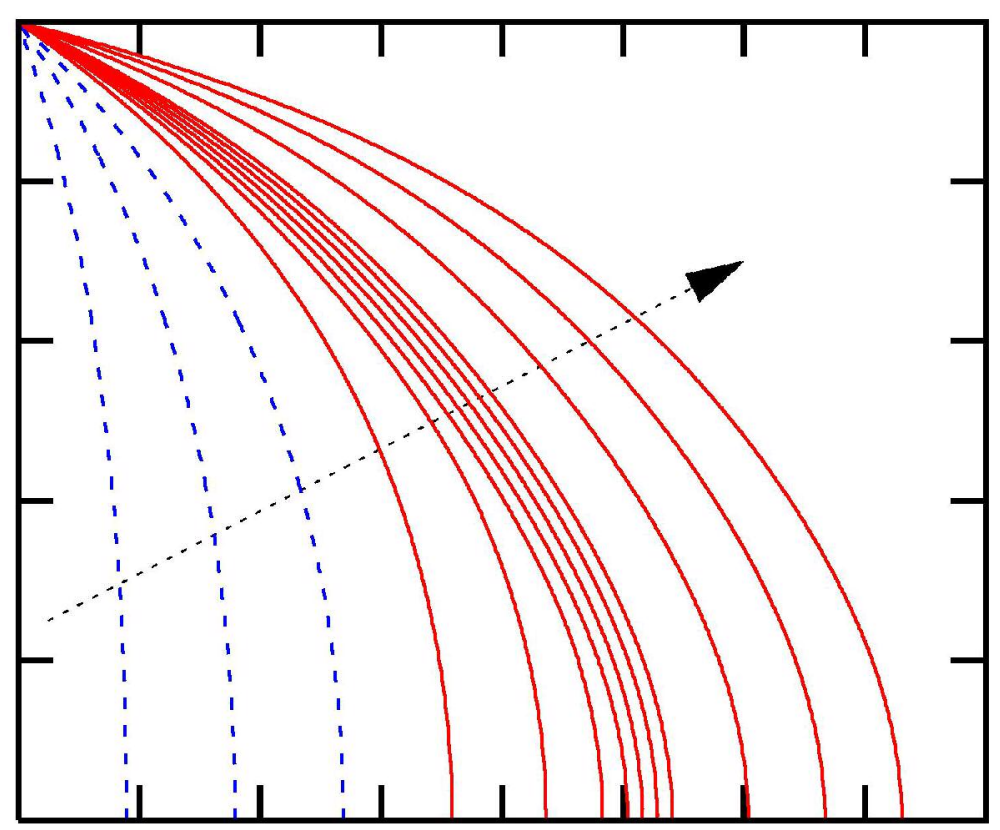

$82 \times 64 \mathrm{~mm}(600 \times 600 \mathrm{DPI})$ 


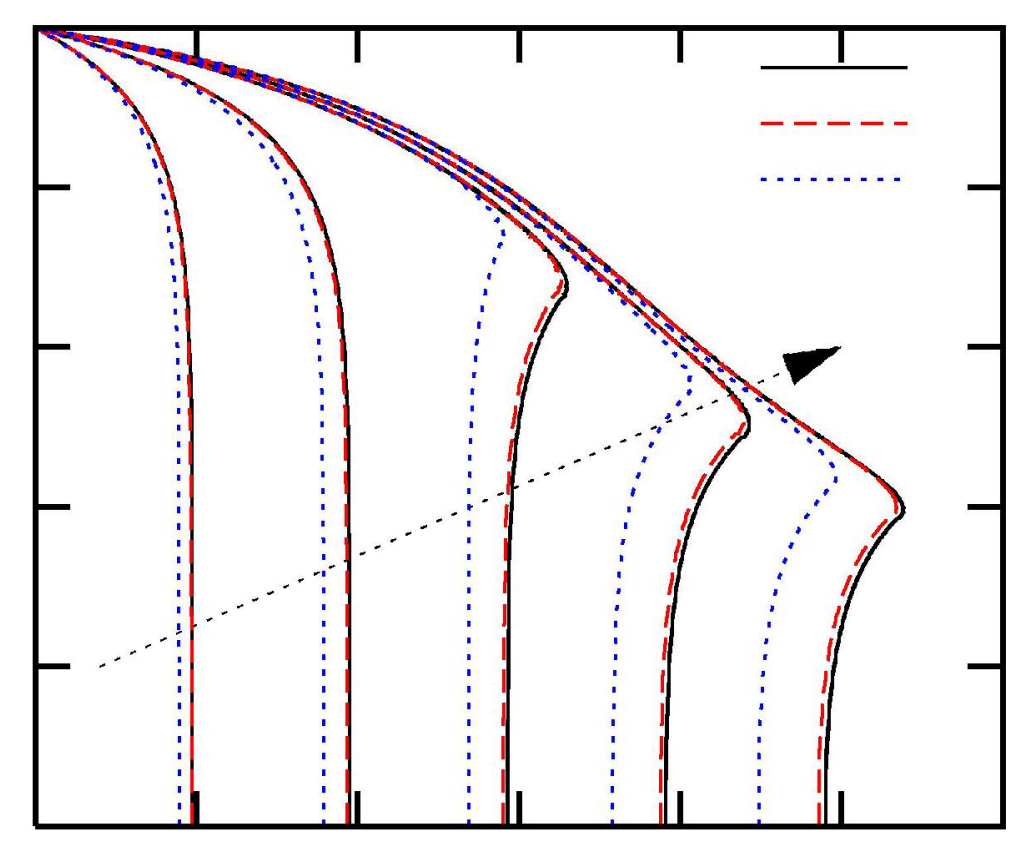

$82 \times 64 \mathrm{~mm}(600 \times 600$ DPI $)$ 


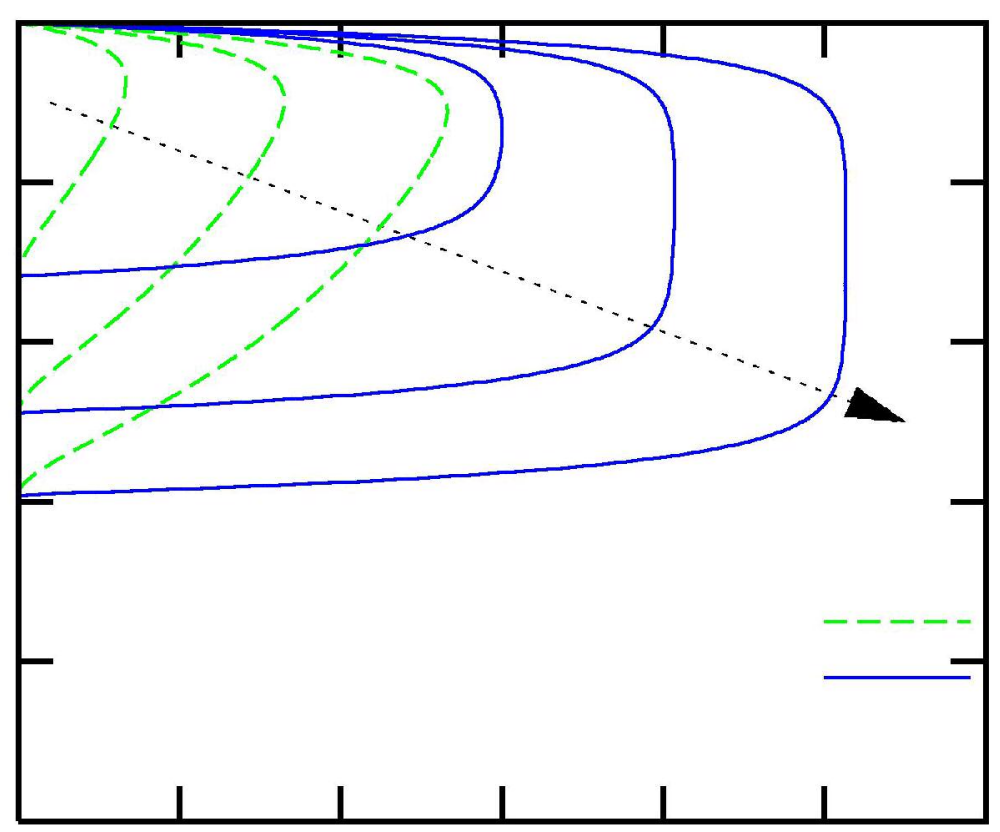

$82 \times 64 \mathrm{~mm}(600 \times 600 \mathrm{DPI})$ 